Prepared for the U.S. Department of Energy

under Contract DE-AC05-76RL01830

\title{
Viscoelastic Model for Lung Parenchyma for Multi-Scale Modeling of Respiratory System Phase II: Dodecahedral Micro-Model
}
AD Freed
JP Carson
DR Einstein
RE Jacob

March 2012

\section{Pacific Northwest}

NATIONAL LABORATORY

Proudly Operated by Battelle Since 1965 


\title{
DISCLAIMER
}

This report was prepared as an account of work sponsored by an agency of the United States Government. Neither the United States Government nor any agency thereof, nor Battelle Memorial Institute, nor any of their employees, makes any warranty, express or implied, or assumes any legal liability or responsibility for the accuracy, completeness, or usefulness of any information, apparatus, product, or process disclosed, or represents that its use would not infringe privately owned rights. Reference herein to any specific commercial product, process, or service by trade name, trademark, manufacturer, or otherwise does not necessarily constitute or imply its endorsement, recommendation, or favoring by the United States Government or any agency thereof, or Battelle Memorial Institute. The views and opinions of authors expressed herein do not necessarily state or reflect those of the United States Government or any agency thereof.

\author{
PACIFIC NORTHWEST NATIONAL LABORATORY \\ operated by \\ BATTELLE \\ for the \\ UNITED STATES DEPARTMENT OF ENERGY \\ under Contract DE-AC05-76RL01830
}

Printed in the United States of America
Available to DOE and DOE contractors from the Office of Scientific and Technical Information,
P.O. Box 62, Oak Ridge, TN 37831-0062;
ph: (865) 576-8401
fax: $(865)$ 576-5728
email: reports@adonis.osti.gov

\begin{abstract}
Available to the public from the National Technical Information Service, U.S. Department of Commerce, 5285 Port Royal Rd., Springfield, VA 22161 ph: (800) 553-6847 fax: $(703) 605-6900$ email: orders@ntis.fedworld.gov online ordering: http://www.ntis.gov/ordering.htm
\end{abstract}

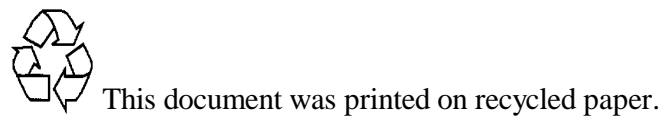




\title{
Viscoelastic Model for Lung Parenchyma for Multi-Scale Modeling of Respiratory System Phase II: Dodecahedral Micro-Model*
}

\begin{abstract}
Alan D. Freed ${ }^{\dagger}$
Clifford H. Spicer Chair in Engineering College of Science, Engineering \& Technology Saginaw Valley State University 202 Pioneer Hall, 7400 Bay Road University Center, MI 48710 USA

E-Mail: adfreed@svsu.edu

Daniel R. EINSTEIN

JAMES P. CARSON

RichaRd E. JACOB

Pacific Northwest National Laboratory 902 Battelle Boulevard P.O. Box 999, MSIN P7-58

Richland, WA 99352 USA

E-Mail: daniel.einstein@pnl.gov james.carson@pnl.gov richard.jacob@pnl.gov
\end{abstract}

March 30, 2012

\begin{abstract}
In the first year of this contractual effort a hypo-elastic constitutive model was developed and shown to have great potential in modeling the elastic response of parenchyma [19, 20]. This model resides at the macroscopic level of the continuum. In this, the second year of our support, an isotropic dodecahedron is employed as an alveolar model. This is a microscopic model for parenchyma. A hopeful outcome is that the linkage between these two scales of modeling will be a source of insight and inspiration that will aid us in the final year's activity: creating a viscoelastic model for parenchyma.
\end{abstract}

\section{Introduction}

The literature on modeling of lung parenchyma is quite substantial, and goes back at least a century and a half [48]. The well-established

${ }^{*}$ The project described was supported by Award Number 5R01HL073598 from the National Heart, Lung, and Blood Institute to Pacific Northwest National Laboratory. The content is solely the responsibility of the authors and does not necessarily reflect the official views of the National Heart, Lung, and Blood Institute or the National Institutes of Health.

${ }^{\dagger}$ Second annual report to Pacific Northwest National Laboratory from Saginaw Valley State University reporting on progress made under grant number 136492. 
non-linearity of the pressure/volume response of lung has made work in this field challenging [30]. Early attempts have, for example, compared the response of lung to finite elasticity via the stretching of a balloon [32]. But most studies, both theoretical and experimental, e.g., $[29,41]$, have applied the classical theory of linear elasticity (which is only applicable for infinitesimal strains and rotations) to neighborhoods surrounding what are considered to be quasi-static states of uniform pressure, which are taken as individual reference configurations. Literature reviews in lung mechanics include: FAFFe \& Zin [12], Fredberg \& Kamm [14], Fung [24, 25], Mead [45], Otis [48, 49], Stamenović [57], Suki et al. [60, 61], TschumperLin et al. [66], Weibel \& Gil [68], and West [69].

Before embarking, it is useful to define some basic concepts. Of primal importance to lung mechanics is the transpulmonary pressure $P$, which is the inflation pressure acting across the lung. It is the difference between alveolar pressure (approximately atmospheric pressure) and pleural pressure. Pleural pressure arises as the stress of suction imposed upon the external pleural membrane through its fluidic adhesion to the ribcage. The volume of the thoracic cavity changes by contractions occurring in the diaphragm and the intercostal muscles of the ribcage [14, 48, 69].

\subsection{Methodology}

The approach adopted in this study of the mechanics of lung parenchyma has a different theoretical underpinning than is typically considered, viz., the finite deformation theory known as hypo-elasticity [64]. In the first annual report for this project [19], the authors derived a hypo-elastic theory for parenchyma, whose findings are published in [20]. The resulting model is characterized by four material parameters: the two LAMÉ constants, $\lambda$ and $\mu$, and their hypo-elastic analogs, $\alpha$ and $\beta$, which are the so-

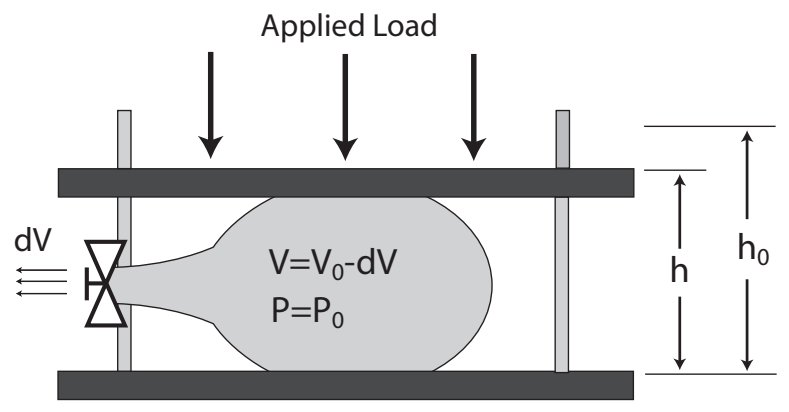

Figure 1: Schematic of the experimental setup of LaI-Fook et al. [40] taken from Ref. [20, Fig. 4].

called Fung constants. ${ }^{1}$ These four parameters can be characterized by a fairly straightforward experiment whose fixturing is drawn in Fig. 1, and whose protocol is described below.

1. Inflate an excised lobe of lung to its total lung capacity (TLC, $\sim 30 \mathrm{~cm} \mathrm{H}_{2} \mathrm{O}^{2}$, the volume where pressure plateaus) and allow it to equilibrate there.

2. Slowly deflate the lobe to a pre-specified pressure, e.g., $P_{\Omega}=20 \mathrm{~cm} \mathrm{H}_{2} \mathrm{O}$, and allow it to equilibrate there.

3. Perform a small pressure cycle $\pm \mathrm{d} P$ from this pressure/volume state $\left(P_{\Omega}, V_{\Omega}\right)$, thereby causing a small change in volume $\mathrm{d} V$ to occur. Finish by cycling up to TLC, and slowly deflating back down to $P_{\Omega}$.

4. Apply a small compressive load $\mathrm{d} f$ to the lobe of lung while maintaining a constant pressure $P_{\Omega}$ within, as illustrated in Fig. 1. Record the applied perturbation in axial stress $\mathrm{d} \sigma=\mathrm{d} f / A_{\Omega}$, with $A_{\Omega}$ denoting the cross-sectional area of the lobe at state $\left(P_{\Omega}, V_{\Omega}\right)$. Measure the corresponding axial stretch $\lambda=h / h_{0}$ and its displacement $\mathrm{d} \lambda=\left(h-h_{0}\right) / h_{0}$.

1. Y.-C. Fung did not derive the hypo-Fung solid; FREED [18] did. Rather, his [22] experiments inspired it.

2. Unit conversions: $1 \mathrm{~Pa}=1 \mathrm{~N} / \mathrm{m}^{2}=10 \mathrm{dyn} / \mathrm{cm}^{2}$ $=0.0102 \mathrm{~cm} \mathrm{H}_{2} \mathrm{O}=0.0075 \mathrm{~mm} \mathrm{Hg}$. 
5. Repeat this process at sequentially lower states of reference pressure $P_{\Omega}$.

\subsubsection{Hypo-Elastic Predictions}

Three, elastic, tangent responses have been established by applying this experimental protocol to our hypo-elastic material model for parenchyma [19, 20].

The first is a measure of the tissue's shape response, where $\mu$ is the classic shear modulus from the linear theory of elasticity. This effective shear modulus is described by [20, Eq. 1]

$$
\tilde{\mu} \doteq \frac{\mathrm{d} \sigma}{\mathrm{d} \lambda / \lambda}=2(\mu+\beta P),
$$

where $\mathrm{d} \sigma$ and $\mathrm{d} \lambda$ are both negative valued, while $P$ is positive valued in LAI-FooK's experiment.

The second tangent response is a measure of the tissue's dilatation, where $\kappa=\lambda+\frac{2}{3} \mu$ is the classic bulk modulus from the linear theory of elasticity. This effective bulk modulus is described by [20, Eq. 48]

$$
\tilde{\kappa} \doteq \frac{\mathrm{d} P}{\mathrm{~d} V / V}=\hat{\kappa}+\left(\alpha+\frac{2}{3} \beta-1\right) P,
$$

where $\hat{\kappa}=|\mathbf{F}|^{-1} \kappa=V_{0} \kappa / V$ represents the bulk modulus of the reference state, viz., $\kappa$, pushed forward into the current state as $\hat{\kappa}$, with $V_{0}$ denoting the lung's volume in this initial reference state, viz., at zero transpulmonary pressure, i.e., at $P=0$. The minus one arises from the fact that volume change accompanies pressure change in Step 3 of this experimental protocol, from which the above relationship follows.

The third tangent response is a measure of the tissue's deformation covariance. This effective Porsson's ratio is given by [20, Eq. 51]

$$
\begin{aligned}
& \tilde{v} \doteq-\frac{\mathrm{d} \lambda_{t} / \lambda_{t}}{\mathrm{~d} \lambda / \lambda} \\
&=\frac{1}{2} \frac{3 \hat{\kappa}+3 \alpha(P+\mathrm{d} \sigma / 2)+2 \beta(P+\mathrm{d} \sigma)}{3 \hat{\kappa}+3 \alpha(P-\mathrm{d} \sigma / 4)+2 \beta(P-\mathrm{d} \sigma / 2)},
\end{aligned}
$$

where $\lambda_{t}$ is the transverse stretch. Recall that $P$ is positive valued, while $\mathrm{d} \sigma$ is negative valued in a LAI-FooK experiment; consequently, this PoIsson's tangent ratio $\tilde{v}$ starts out at a half, and diminishes slowly, in a pressure dependent way, as the traction $|\mathrm{d} \sigma|$ increases.

These predictions only apply for the BVP that is the LaI-FooK et al. [40] experiment, which is described in the above protocol.

\subsubsection{Experimental Validation}

Data acquired from experiments described by this BVP can be found in the literature, e.g., $[40,58,59]$. From these and other published data, one finds that $\beta=O(1)$ and $\alpha=O(10)$ for parenchyma. Hypo-elasticity predicts a linear dependence with respect to transpulmonary pressure for both the shear and bulk responses. Such behaviors do not have to be artificially introduced via, e.g., pressure dependent moduli, as is usually done, cf. e.g., with [10].

The linear prediction of Eq. (1) for describing the shear response in lung agrees very well with experimental observations [19], as shown in Fig. 2. The validity of Eq. (1) requires the tangent modulus $\tilde{\mu}=\mathrm{d} \sigma /(\mathrm{d} \lambda / \lambda)$ to be measured from incremental excursions according to Step 4 in the above protocol.

In stark contrast with Eq. (1), the linear prediction of Eq. (2) for describing the bulk response of lung, measured from incremental pressure/volume excursions according to Step 3, does not capture the whole essence of the measured bulk response of lung. This is apparent in the experimental data of Stamenović \& Yager [59, Fig. 2] redrawn here in Fig. 3. Equation (2) does capture the first-order nature of this response quite nicely, viz., an increasing bulk stiffness with increasing pressure, which is a prediction that classical elastic formulations cannot make. However, Eq. (2) is incapable of predicting the second-order excursions from linearity that prominently appear in Fig. 3. STA- 


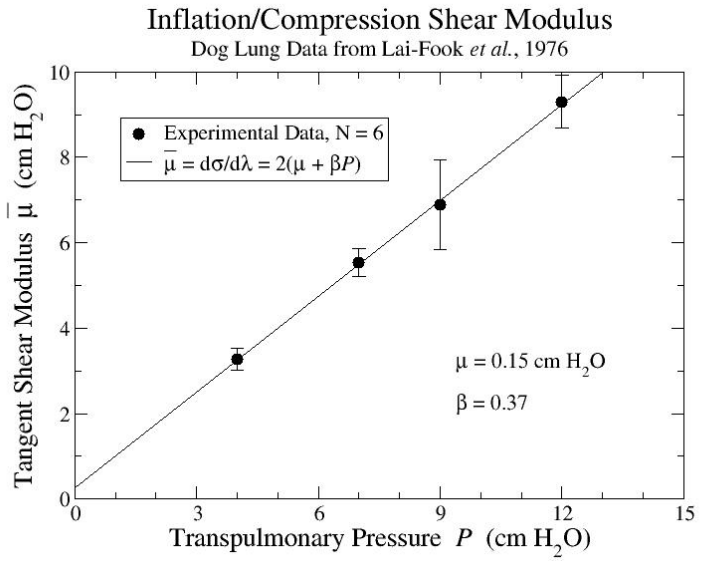

Figure 2: A least squares fit of Eq. (1) for experimental data taken from LAI-Fook et al. [40, Fig. 3], with an $R^{2}$ error of 0.9990. Error bars are \pm one standard deviation in sample error.

MENOvić \& YAGER report that the idiosyncrasies of this response likely reside with the affect that surface tension has on dilatation, and they offered a solution methodology that addresses this point.

A further examination of Fig. 3 implies that the mechanics of breathing may actually

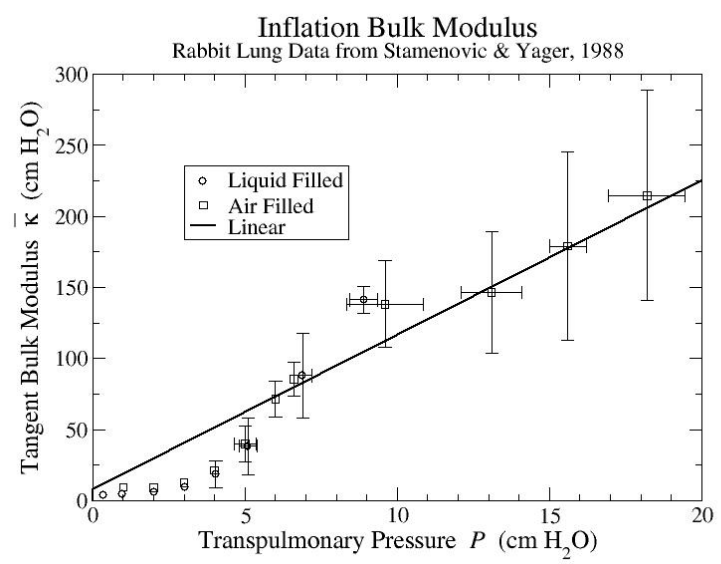

Figure 3: Experimental data of Stamenović \& YAGer [59, Fig. 2]. Error bars are \pm one standard deviation in sample error. The straight line is indicative of Eq. (2). Deviations from this line are caused by the surfactant cycle.

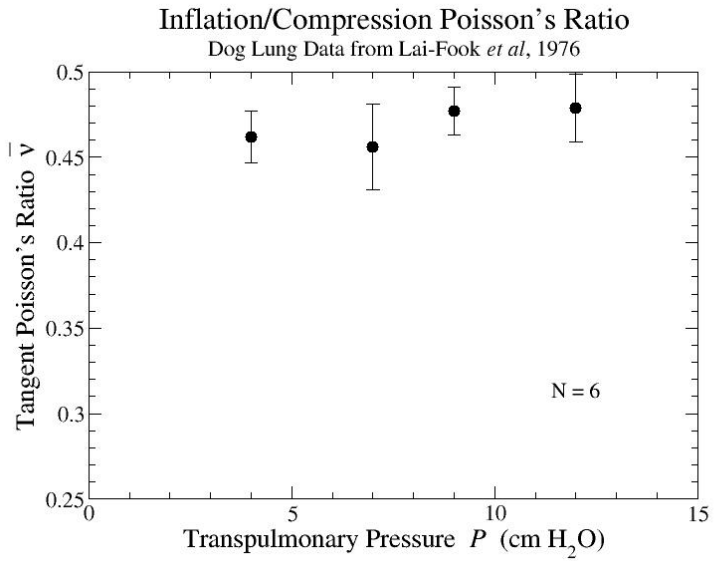

Figure 4: Experimental data extracted from LAI-Fook et al. [40, Fig. 3]. Error bars are \pm one standard deviation in sample error.

dissipate less energy than had previously been thought. This can be seen in the departure of the data from the curve of Eq. (2). The elastic response from classical theory is a horizontal line in Fig. 3, while the hypo-elastic theory predicts a sloped line, which is more representative of the data. Deviations between elastic (non-dissipative) predictions and experimental data are typically interpreted as being caused by dissipative processes. This has a physical soundness to it in that Nature tends to prefer biological designs that conserve as much energy as is possible.

Theory predicts, for the LAI-Fook BVP, that the local, effective, PoIsson's ratio will be somewhere near to a $1 / 2$. Validation of this expectation is presented in Fig. 4. The PoIsson's ratio applicable to this BVP is very different from the one that arises from the simple extension of a uniform rod, which is given by $\tilde{v}=v+\alpha \sigma / 4(\lambda+\mu)$ [20, Eq. 35], where $\sigma$ is the true stress, and $v=\lambda / 2(\lambda+\mu)$ is the PoIsson's ratio from classical elasticity theory. 


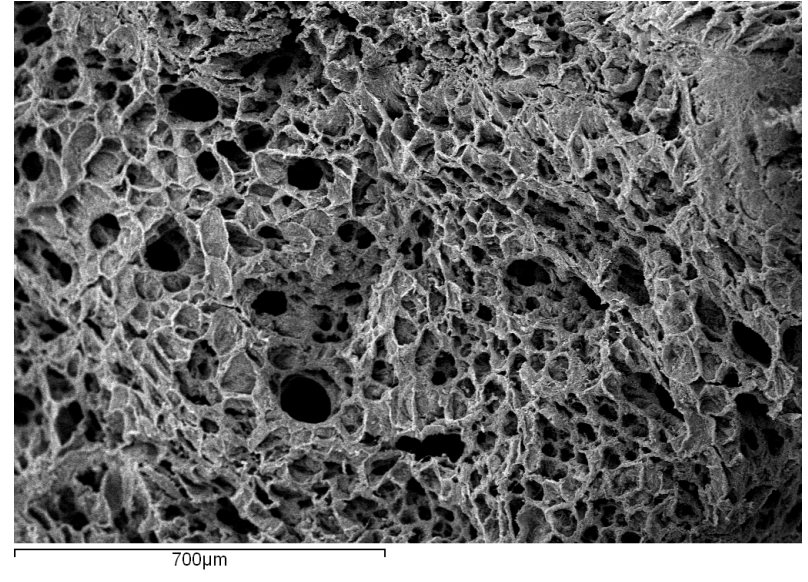

Figure 5: SEM photograph of rat lung.

\subsection{Objective}

To gain an understanding about how one ought to proceed in augmenting our hypo-elastic continuum model to better describe reality we seek, in this report, insight and inspiration through a micro-mechanical model, where the physical effects that control alveolar response are thought to be more faithfully represented.

\section{Structure of Lung}

SEM photographs of rat lung, taken by Prof. S. SHEPARDSON at SVSU over a range in magnifications, are provided in Figs. 5-8. ${ }^{3}$

In Fig. 5, the parenchyma is seen to be a fairly uniform sea of tiny alveolar sacks that are periodically perforated by alveolar ducts for transporting the air into and out of the lung. This photo focuses on a roughly $1 \mathrm{~mm} \times 1 \mathrm{~mm}$ sectioning of lung.

In Fig. 6, zooming in on this alveolar landscape, while focusing one's attention on the forth quadrant in the photo, one observes that the alveolar mouths actually comprise a large area fraction of the wall dimension of alveolar ducts [68]. These airways are highly perforated.

Continuing to zoom in, in the central region of Fig. 7, one sees the mouths of several alveo-

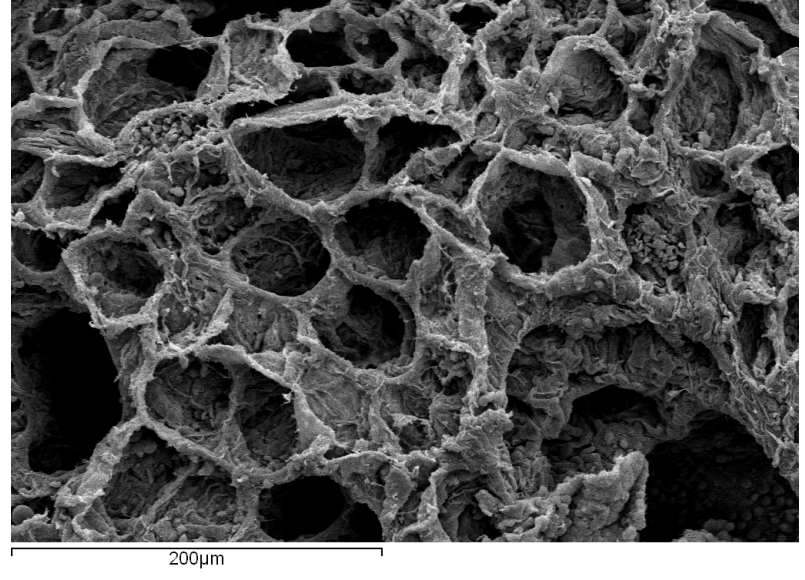

Figure 6: SEM photograph of rat lung.

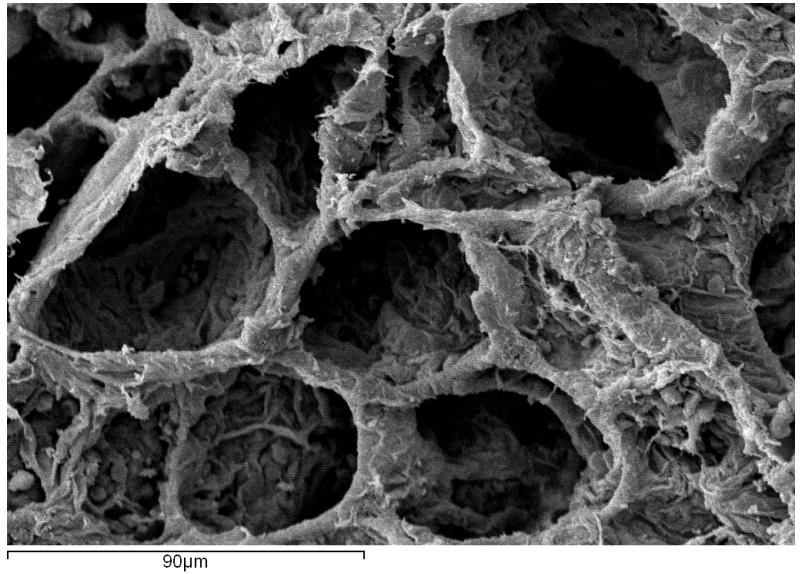

Figure 7: SEM photograph of rat lung.

lar sacs. These annuli have pentagonal shapes, and are comprised of heavier cords of fiber than are found elsewhere throughout their structures [44]. The pentagonal facets that are the alveolar walls are shared membranes between two neighboring alveoli. Random fiber filaments (elastin and collagen) are seen to thread from

3. Protocol for SEM sample preparation for rat lung tissue: Primary fixation consisted of $2.5 \%$ glutaraldehyde in a $0.1 \mathrm{M}$ phosphate buffer and $1.0 \%$ sucrose $+\mathrm{CaCl}_{2}, \mathrm{pH} 7.2$, at $4^{\circ} \mathrm{C}$ for 2 hr. Post fixation was with $1.0 \% \mathrm{OsO}_{4}$ in buffer for $1 \mathrm{hr}$. at $4^{\circ} \mathrm{C}$. Both fixation steps were followed by 4 buffer washes at 15 min. each. Dehydration was in a graded series of acetone. Samples were critical point dried (Denton DCP-1), sputter coated with gold (Denton Desk II) twice at $40 \mathrm{mAmp}$ and 50 Torr for $2 \mathrm{~min}$., and then viewed with the JEOL 5400 SEM at $15 \mathrm{kV}$. 


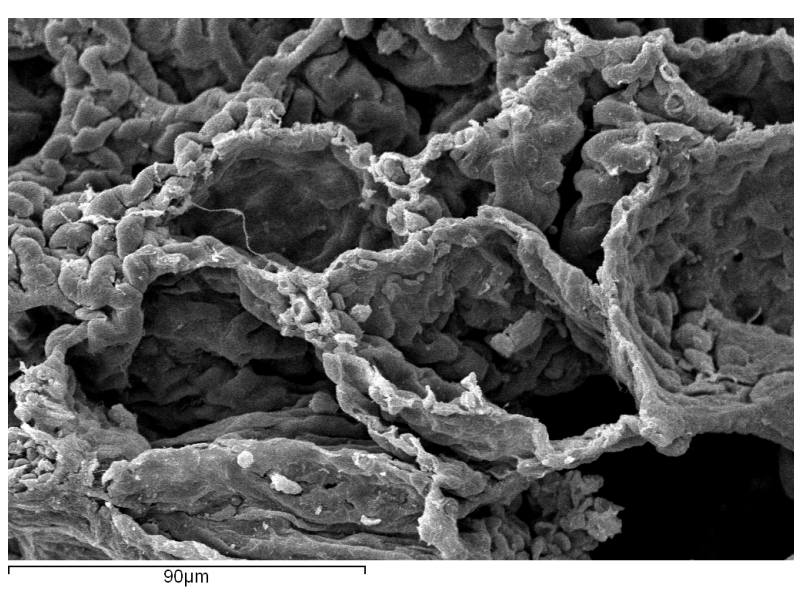

Figure 8: SEM photograph of rat lung.

the septa, crisscrossing their faces [56].

The final photograph, shown in Fig. 8, and taken at the same magnification as Fig. 7, does a nice job of showing the capillary network that intertwines with the fibrous structure of the alveolar walls [33]. This is especially visible in the second quadrant of the image. It is here that the gas exchange between air and blood takes place via diffusion across the capillary walls [68, 69]. All evidence of a coating layer of surfactant has been removed by the sample preparation procedure.

It is photographs like these that provide impetus for the following micro-modeling effort.

\section{Dodecahedral Model}

"Constitutive equations are phenomenological. They are regarded as empirical by experimenters, and axiomatic by mathematicians. In biomechanics, we often try to derive them on the basis of microstructure $\ldots$ in order to gain a better understanding, or to get some guidance to the mathematical form."

FUNG [24,pg. 431]

This is the roadmap adopted here.
Typical alveoli are fourteen sided polyhedra with one face being open as its mouth to an alveolar duct [33]. To capture the microstructural features of lung, researchers have modeled both alveoli and alveolar ducts. Two different geometric shapes are usually employed when modeling an alveolus: a dodecahedron, as introduced by Frankus \& LeE [13]; and a truncated octahedron, as introduced by Dale, Matthews \& Schroter [6]. A dodecahedron is a twelve sided polyhedron with each face being a regular pentagon. A truncated octahedron is a pair of regular pyramids stacked bottom to bottom, which is an octahedron, whose six points are then lopped off. This produces a fourteen sided polyhedron with six faces that are squares and eight faces that are hexagons, where like shapes have like dimensions.

The truncated octahedron is volume filling. It is therefore the preferred geometry to use whenever one sets out to construct assemblages of alveoli to build a microstructural model that is to be solved numerically via a finite element method. The purpose of such an exercise is to homogenize the response of an assemblage of alveoli up to the macroscopic level, i.e., the level of a continuum mass point, viz., the parenchyma [6, 7, 8, 9, 39].

The dodecahedron is an isotropic structure. It is therefore the preferred geometry to use whenever a single alveolus is to be used as a representative volume element (RVE) to be homogenized, in closed form, up to the macroscopic level [4, 36, 37]. Here the isotropy of the microstructure ensures an isotropic macro response. Parenchyma, as a tissue, is isotropic $[23,34]$; whereas, lung, as an organ, is a complex, heterogeneous structure [45, 69]. (This can be seen, e.g., in Fig. 14.) This distinction has, from time-to-time, lost focus in the literature, cf. e.g., [10]. For the reasons stated above, a dodecahedron, drawn in Fig. 9, is the geometric structure selected for use in this study. 


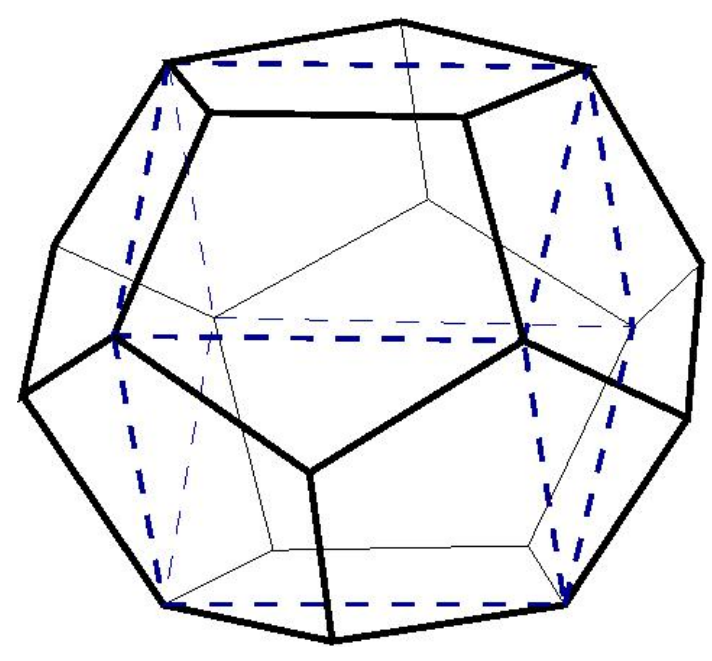

Figure 9: Geometric representation of a dodecahedron. A cube is contained within a dodecahedron, with one of its five possible orientations being displayed. This geometry was introduced by Frankus \& LeE [13], and later adopted by Kimmel et al. [4, 36, 37], for the purpose of approximating an alveolar structure in their mathematical modelings of lung parenchyma.

Only one-third of the cross-sectional area of a septal fiber, and only one-half of the alveolar wall thickness, associate with any given dodecahedron. Specifically, one-third of the total force carried by a septal fiber associates with a given alveolus, with the remaining two-thirds of the transmitted force belonging to the two adjoining alveoli. Likewise, half of the surface traction carried along a membranous wall associates with a given alveolus, with the other half of its surface traction belonging to its adjacent alveolus.

Our ultimate objective is to develop a computationally efficient continuum model for lung parenchyma for use as a material model within a finite volume CFD code, specifically,

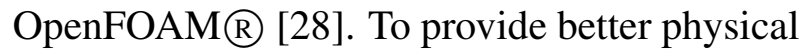
interpretations to such a model's parameters, a micro-mechanical model is sought. Specifically, the analytical approach of KIMMEL and his colleagues is selected over the numerical approach of ScHROTER and his colleagues for our purpose: homogenization of an RVE.

\subsection{Geometric Scalings}

In terms of the spetal length $L$, which associates with the length of one side of a pentagon, the area of a regular pentagon in a dodecahedron is

$$
\begin{aligned}
A & =\frac{5}{4} \tan (\omega) L^{2} \\
& \approx 1.720 L^{2},
\end{aligned}
$$

where the inside angles of a regular pentagon all measure $108^{\circ}=2 \omega$, i.e., the sum of two inside angles belonging to any neighboring pair of the five, enclosed, isosceles triangles, each with an angle $\omega$. Utilizing the dodecahedral dimensions illustrated in Budiansky \& Kimmel [4, Fig. 2], the distance across a pentagon is

$$
\begin{aligned}
D & =\frac{1}{2}(\tan (\omega)+\sec (\omega)) L \\
& \approx 1.539 L,
\end{aligned}
$$

where scaling factors have been truncated at four significant figures.

The volume of a dodecahedron is

$$
\begin{aligned}
V & =5 \tan ^{2}(\omega) \sin (\omega) L^{3} \\
& =4 \tan (\omega) \sin (\omega) A L \\
& \approx 7.663 L^{3} .
\end{aligned}
$$

The area of a dodecahedron projected onto a plane containing one of the pentagonal faces is

$$
\begin{aligned}
\bar{A} & =5 \tan (\omega) \sec (\omega) \cos (\alpha) L^{2} \\
& =4 \sec (\omega) \cos (\alpha) A \\
& \approx 11.14 L^{2}
\end{aligned}
$$

where $\alpha=\pi / 20=18^{\circ}$. (There are twenty, equal, pie-shaped wedges that comprise this projected area.) The nominal diameter of this area is

$$
\begin{aligned}
\bar{D} & =\tan (\omega)(1+\cos (\alpha)) L \\
& \approx 2.685 L,
\end{aligned}
$$


which is the average of the shortest and longest diameters across this plane of projection.

All of the above geometric values have been expressed in terms of the septal boundary length, i.e., the length along one side of a pentagon whose self-similar patterning is the generator for a dodecahedron's surface. As a matter of notation, whenever a dimension applies to both geometries, e.g., a diameter, the dodecahedral dimension will have a bar placed over it; whereas, the pentagonal dimension will not.

To dimension the alveoli of human lung, Sobin, Fung \& Tremer [56] measured the mean chord lengths, viz., $\bar{D}$, across individual alveoli sectioned from lungs that were fixed at different pressures. Samples were taken from 9 lungs extracted postmortem from individuals between 16 to 89 years of age. ${ }^{4}$ At a transpulmonary pressure of $4 \mathrm{~cm} \mathrm{H}_{2} \mathrm{O}$, the measured mean chord length from 1423 distinct measurements was $\bar{D}=191 \pm 86 \mu \mathrm{m}$; at a pressure of $10 \mathrm{~cm} \mathrm{H}_{2} \mathrm{O}, \bar{D}=202 \pm 88 \mu \mathrm{m}$ from 1296 measurements; and at a pressure of $14 \mathrm{~cm} \mathrm{H}_{2} \mathrm{O}$, $\bar{D}=235 \pm 99 \mu \mathrm{m}$ from 1083 measurements. These data are plotted in Fig. 10. All reported and drawn error bounds pertain to plus/minus one standard deviation in the measurements.

\subsection{Model Assumptions}

Following procedures put forward by BudianSKY \& KimMEL [4, 36], virtual displacements are imposed onto a dodecahedron. By generalizing their analysis, the constitutive responses have been left general, instead of being given a specific description, as in their works. Boundary value problems are solved via the principle of virtual work to hopefully reveal, in our case, equivalent hypo-elastic moduli that correspond to such a stimulated continuum.

A microscopic modeling of parenchyma is put forward that builds upon the following list

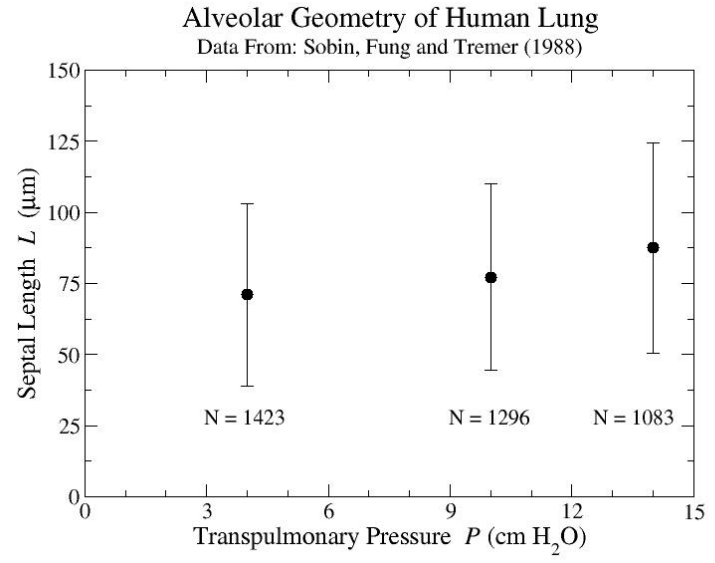

Figure 10: Septal length $L$ as a function of transpulmonary pressure $P$ in human lung. The alveolar diameters $\bar{D}$ and their standard deviations in error, as reported by SoBIN et al. [56], have been converted into measures of septal length $L$ via Eq. (8).

of assumptions advanced by KIMMEL and his colleagues, cf. [4, 37]:

- A regular, dodecahedral, space truss represents the geometry of an alveolus, in an averaged sense.

- All truss elements are pinned (carry no moments) and remain in tension.

- The alveolar mouth, with its thicker fibers and open face, is modeled with a phantom face, and fibers sized like any of the other eleven pentagonal elements comprising the dodecahedron. ${ }^{5}$

4. SoBIN et al. [56] documented an age effect in these data that has been averaged over here, i.e., ignored.

5. Kimmel \& Budiansky [36] address this point via a private communication they had with Prof. T. A. WILson; specifically:

"Professor T. A. WILson notes that the present model does not take explicit account of either alveolar openings or their fibrous boundaries. WILson suggests that the elastic resistance of the ring boundaries tends to make up for the 
- The geometry remains self-similar whenever it is subjected to an isotropic state of stress/pressure.

\subsection{Bulk Response}

Following the method of analysis laid out by Budiansky \& Kimmel $[4,36]$, which is in accordance with Step 3 of the LAI-FooK experimental protocol found on pg. 2, the principle of virtual work is applied to a dodecahedral space truss of volume $V_{\Omega}$ loaded to a state of hydrostatic (or transpulmonary) pressure $P_{\Omega}$ belonging to reference state $\Omega$. By imposing their condition of self-similarity, it follows that identical axial forces of traction $F_{\Omega}$ exist in all 30 of its members of length $L_{\Omega}$, plus there are identical equi-biaxial forces of traction $T_{\Omega}$ that exist across all 12 of its faces of area $A_{\Omega}$ so that

$$
P_{\Omega} \delta V=30 F_{\Omega} \delta L+12 T_{\Omega} \delta A .
$$

Furthermore, self-similar growth of a dodecahedron under increasing pressure $P_{\Omega}$ requires, via Eqs. (4 \& 6), that

$$
\frac{\delta V}{V_{\Omega}}=3 \frac{\delta L}{L_{\Omega}}=\frac{3}{2} \frac{\delta A}{A_{\Omega}},
$$

so that, when combined with Eq. (9), one gets

$$
P_{\Omega} V_{\Omega}=10 F_{\Omega} L_{\Omega}+8 T_{\Omega} A_{\Omega} .
$$

From Eqs. (4 \& 6), this predicts a hydrostatic alveolar pressure of

$$
P_{\Omega}=\frac{8 \cos (\omega)}{\phi^{2} L_{\Omega}}\left(\frac{2 \cos (\omega)}{\phi L_{\Omega}} F_{\Omega}+T_{\Omega}\right),
$$

where $\phi=2 \sin (\omega)$ is the so-called golden ratio. This is an equilibrium force balance. It is therefore independent of any specific constitutive behaviors that enter as functions describing the axial force $F\left(L_{\Omega}\right)$ or the surface traction $T\left(L_{\Omega}\right)$, wherein the septal length $L_{\Omega}$ is the independent variable.

\subsubsection{Modulus}

Differentiating Eq. (11) gives

$$
\begin{aligned}
V_{\Omega} \mathrm{d} P & +P_{\Omega} \mathrm{d} V \\
= & 10\left(L_{\Omega} \mathrm{d} F+F_{\Omega} \mathrm{d} L\right) \\
& +8\left(A_{\Omega} \mathrm{d} T+T_{\Omega} \mathrm{d} A\right),
\end{aligned}
$$

from which a local (tangent) bulk modulus can be defined, as in Eq. (2), via

$$
\tilde{\kappa}=\left.\frac{\mathrm{d} P}{\mathrm{~d} V / V}\right|_{\Omega},
$$

so that, upon combining Eqs. $(9,10$ \& 13) with Eq. (14), one is lead to

$$
\begin{aligned}
\tilde{\kappa}=10 & \left.\left(\frac{\mathrm{d} F}{\mathrm{~d} L / L}-2 F\right)\right|_{\Omega} \frac{\mathrm{d} L}{\mathrm{~d} V} \\
& +\left.8\left(\frac{\mathrm{d} T}{\mathrm{~d} A / A}-\frac{T}{2}\right)\right|_{\Omega} \frac{\mathrm{d} A}{\mathrm{~d} V} .
\end{aligned}
$$

With the aid of Eqs. (4, $6 \& 10)$, the above expression can be rewritten as

$$
\begin{aligned}
\tilde{\kappa}=\frac{16 \cos (\omega)}{3 \phi^{2} L_{\Omega}} & \left\{\left.\frac{\cos (\omega)}{\phi L_{\Omega}}\left(\frac{\mathrm{d} F}{\mathrm{~d} L / L}-2 F\right)\right|_{\Omega}\right. \\
& \left.+\left.\left(\frac{\mathrm{d} T}{\mathrm{~d} A / A}-\frac{T}{2}\right)\right|_{\Omega}\right\} \cdot
\end{aligned}
$$

Like Eq. (12), Eq. (16) is independent of one's choice for constituent response functions describing a dodecahedron's individual material elements.

This micro-model has a direct macro-model analog in Eq. (2), and therefore is a useful point of entry to study a potential micro-macro linkage.

\subsection{Axial Response}

Here the BVP of the LaI-Fook et al. [40] experiment, illustrated in Fig. 1 and described on missing surface tension in the holes, so that neglect of both effects may be selfcompensating." 


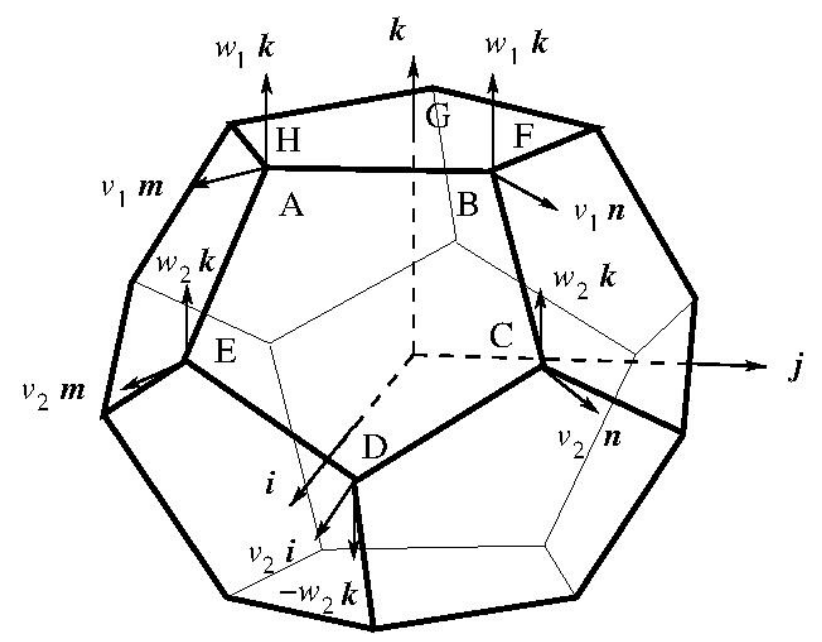

Figure 11: A dodecahedron subjected to a uni-directional load applied in the $\mathbb{k}$ direction, which can be described in terms of a set of four displacements $\left\{w_{1}, v_{1}, w_{2}, v_{2}\right\}$.

pg. 2, is imposed upon a dodecahedral truss in accordance with the geometric analysis of Budiansky \& Kimmel [4], which is illustrated in Fig. 11.

It is supposed that an external traction $\sigma$, acting in the $k$ direction, is applied to the twelve planar faces of a dodecahedron, as oriented in Fig. 11. Furthermore, it is supposed that this dodecahedron was previously subjected to a hydrostatic pressure of $P_{\Omega}$ that serves as its state of reference, with $L_{\Omega}$ denoting the length of each truss element in this isotropic reference configuration $\Omega$.

In the $\left(P_{\Omega}, L_{\Omega}\right)$ reference state affixed to the $(i, j, k)$ Cartesian frame, as depicted in Fig. 11, one determines that the coordinates for nodes $A, B, C, D$, and $E$ (see App. A for details) are $^{6}$

$$
\begin{aligned}
A & =\frac{\phi L_{\Omega}}{2}(\phi \sin (\varphi),-1 / \phi, \phi \cos (\varphi)), \\
B & =\frac{\phi L_{\Omega}}{2}(\phi \sin (\varphi), 1 / \phi, \phi \cos (\varphi)) \\
C & =\frac{\phi L_{\Omega}}{2}(\phi \cos (\varphi), 1, \sin (\varphi) / \phi) \\
D & =\frac{\phi L_{\Omega}}{2}(2 \cos (\varphi), 0,-\sin (\varphi) / \phi) \\
E & =\frac{\phi L_{\Omega}}{2}(\phi \cos (\varphi),-1, \sin (\varphi) / \phi)
\end{aligned}
$$

where $\phi=(1+\sqrt{5}) / 2$ is the golden ratio with $\omega=\sin ^{-1}(\phi / 2)=54^{\circ}$ and $\varphi=\tan ^{-1}(1 / \phi) \approx$ $31.72^{\circ}$. The simple set of coordinates put forward in Eq. (17) follow not only from trigonometry, but also from many of the interesting properties of the golden ratio $\phi$ that the geometry of a dodecahedron can be described in terms of, some of which are presented in App. D.

The displacements of nodes $A, B, C, D$, and $E$ from their reference locations in state $\Omega$, as illustrated in Fig. 11, are described by vectors

$$
\begin{aligned}
& \mathbb{u}_{A}=v_{1} m_{n}+w_{1} k, \\
& \mathbb{u}_{B}=v_{1} \mathbb{}+w_{1} k, \\
& \mathbb{u}_{C}=v_{2} \mathbb{m}+w_{2} \mathbb{k}, \\
& \mathbb{u}_{D}=v_{2} i-w_{2} k, \\
& u_{E}=v_{2} m+w_{2} k \text {. }
\end{aligned}
$$

Unit vectors $m$ and $m$, which are directed radially away from $k$, pass through nodes $A, B, C$, and $E$, and are defined by

$$
\begin{aligned}
m & =\sin (\omega) i-\cos (\omega) j, \\
\mathbb{n} & =\sin (\omega) i+\cos (\omega) j,
\end{aligned}
$$

neither of which rotates in space during the deformation of interest, due to the symmetries involved. In other words, the response is radially isotropic within any plane whose normal aligns with the direction of the imposed traction, viz., the $k$ direction, and whose orientation points radially outward, as is the case for $m$ and $\pi$.

6 . This is a different coordinate description from the one used by Budiansky \& Kimmel [4]. 


\subsubsection{Geometric Expansions}

From symmetry of the imposed deformation, there are only three, distinct, line elements, viz., $A B, B C$, and $C D$, with there being ten of each type in the distorted dodecahedron. There are also only two, distinct, area elements, viz., $A B C D E$ and $A B F G H$, with the top and bottom pentagons remaining regular, while all ten of the side pentagons distort from regularity during the assigned loading. It is assumed that:

- The regular planar pentagons of type $A B C D E$ deform into distorted planar pentagons, each composed of an isosceles trapezoid and an isosceles triangle, with line $C E$ being held in common.

This differs from the deformed shape assumed by Budiansky \& Kimmel [4]. They considered the deformed pentagon to be comprised of five distorted triangles that share a common center whose height of displacement is the mean of the five corner displacements.

Budiansky \& Kimmel [4] introduced a set of non-dimensional displacements expressed as $y_{i}$ $(i=1,2,3,4)$ that they defined as

$$
\boldsymbol{y}=\left\{\begin{array}{l}
y_{1} \\
y_{2} \\
y_{3} \\
y_{4}
\end{array}\right\}=\frac{1}{L_{\Omega}}\left\{\begin{array}{l}
w_{1} \\
v_{1} \\
w_{2} \\
v_{2}
\end{array}\right\},
$$

which describe the extensional deformation of a dodecahedron. Their change in variables has been adopted here, too.

The square of the stretch of each of the three, independent, bar elements can be expressed as ${ }^{7}$

$$
\begin{aligned}
& \lambda_{A B}^{2}=1+A_{A B} \cdot \boldsymbol{y}+\frac{1}{2} \boldsymbol{y} \cdot \boldsymbol{A}_{A B} \boldsymbol{y}, \\
& \lambda_{B C}^{2}=1+A_{B C} \cdot \boldsymbol{y}+\frac{1}{2} \boldsymbol{y} \cdot \boldsymbol{A}_{B C} \boldsymbol{y}, \\
& \lambda_{C D}^{2}=1+A_{C D} \cdot \boldsymbol{y}+\frac{1}{2} \boldsymbol{y} \cdot \boldsymbol{A}_{C D} \boldsymbol{y},
\end{aligned}
$$

where $\lambda_{A B}=L_{A B} / L_{\Omega}, \lambda_{B C}=L_{B C} / L_{\Omega}$, and $\lambda_{C D}=L_{C D} / L_{\Omega}$. The $A$ vectors are given in Eq. (87), while the $\boldsymbol{A}$ matrices are given in Eq. (88), both being derived in App. A.

The ratios of areas, viz., the areal stretches of the two, independent, surface elements, can be expressed via series expansions in the displacement vector $y^{8}$

$$
\begin{aligned}
\Lambda_{R}= & 1+\mathbb{B}_{R} \cdot \boldsymbol{y}+\frac{1}{2} \boldsymbol{y} \cdot \boldsymbol{B}_{R} \boldsymbol{y}, \\
\Lambda_{D}= & 1+\mathbb{B}_{D} \cdot \boldsymbol{y}+\frac{1}{2} \boldsymbol{y} \cdot \boldsymbol{B}_{D} \boldsymbol{y} \\
& +O\left(\mathbb{y}^{3}\right),
\end{aligned}
$$

where $\Lambda_{R}=A_{R} / A_{\Omega}$ and $\Lambda_{D}=A_{D} / A_{\Omega}$, with

$$
A_{\Omega}=\frac{5}{4} L_{\Omega}^{2} \tan (\omega)
$$

being the area of each pentagonal face of the dodecahedron in its reference state, while $A_{R}$ and $A_{D}$ are the respective areas of the regular $A B F G H$ and distorted $A B C D E$ pentagon types in their final shapes. The various $\mathbb{B}$ and $\boldsymbol{B}$ arrays are given in Eqs. (89, 91 \& 102), all being derived in App. B. There it is shown that $\boldsymbol{B}_{D}$ is actually a quadratic polynomial in $\boldsymbol{y}$, in which matrix equation (102) is the constant term.

The volume ratio can also be expressed in terms of displacement vector $y$; specifically,

$$
\Delta=1+\mathbb{C} \cdot \boldsymbol{y}+\frac{1}{2} \boldsymbol{y} \cdot \boldsymbol{C} \boldsymbol{y}+O\left(\mathbb{y}^{3}\right),
$$

where $\Delta=V / V_{\Omega}$ with

$$
V_{\Omega}=5 L_{\Omega}^{3} \tan ^{2}(\omega) \sin (\omega)
$$

being the dodecahedron's volume in its reference state. Vector $\mathbb{C}$ is given in Eq. (115), while matrix $\boldsymbol{C}$ is given in Eq. (120), both being derived in App. C.

7. Budiansky \& Kimmel [4] derived an expression for Lagrangian strain. Here, the square of stretch is written in terms of displacements $y$.

8. The surface analysis developed here is substantially different from the areal analysis put forward by Kimmel \& Budiansky [36]. Here, an analytic solution is derived for vectors $\mathbb{B}_{R}$ and $\mathbb{B}_{D}$, and matrices $\boldsymbol{B}_{R}$ and $\boldsymbol{B}_{D}$; whereas, in their paper, a numerical estimate of a Hessian $\boldsymbol{B}$ was used to describe the collective influence of our two matrices $\boldsymbol{B}_{R}$ and $\boldsymbol{B}_{D}$, i.e., they did not distinguish between the pentagons that remain regular from those that distort. 


\subsubsection{RVE Averaged Fields}

In their RVE homogenization of a dodecahedral truss, Budiansky \& Kimmel [4, App. C] derived the following stress potential

$$
\pi=\sigma V_{\Omega} \mathfrak{f} \cdot \not{y},
$$

wherein

$$
f=\left\{\begin{array}{c}
\cos (\varphi) \\
0 \\
\cot (\omega) /(2+\phi) \\
0
\end{array}\right\}
$$

whose components here are expressed in a different form, but are numerically equivalent to those arrived at by Budiansky \& Kimmel. In their analysis, the applied traction $\sigma k$ has units of force per unit area, as measured in the reference state of $\left(P_{\Omega}, L_{\Omega}\right)$. The applied traction $\mathrm{d} \sigma$ in the LAI-Fook experiment will be negative valued, as compressive perturbative loads are being applied.

BudiansKy \& Kimmel volume averaged the dodecahedron's strain response in their App. E, arriving at the following expression for an axial strain increment

$$
\mathrm{d} \epsilon=f \cdot \mathrm{d} y,
$$

i.e., the rate at which work is being done per unit reference volume is $\mathrm{d} \pi / V_{\Omega}=\sigma \mathrm{d} \epsilon$. A local (tangent) modulus can be established from this expression via

$$
\tilde{E}=\frac{\mathrm{d} \sigma}{\mathrm{d} \epsilon}=\frac{\mathrm{d} \sigma}{\mathfrak{f} \cdot \mathrm{d} y},
$$

which is the elastic stiffness at the reference state $\left(P_{\Omega}, L_{\Omega}\right)$. They also derived, in their App. E, a local (tangent) Poisson's ratio of

$$
\begin{aligned}
\tilde{v} & =-\frac{\mathrm{d} \epsilon}{\mathrm{d} \epsilon_{T}} \\
& =-\frac{1}{2}\left(\frac{\phi \mathrm{d} y_{2}+(1+\phi) \mathrm{d} y_{4}}{(1 / 2+\phi) \mathrm{d} y_{1}+\frac{1}{2} \mathrm{~d} y_{3}}\right),
\end{aligned}
$$

written here in terms of the golden ratio $\phi$. Consequently, they compute the effective shear modulus via

$$
\tilde{\mu}=\frac{\tilde{E}}{2(1+\tilde{v})},
$$

according to the classical theory of elasticity.

It follows then that if one knows what the incremental change in displacement $d y$ is, which associates with an imposed increment of traction $\mathrm{d} \sigma$, then one can quantify homogenized values for the effective shear modulus $\tilde{\mu}$ and PoIsson's ratio $\tilde{v}$ that pertain to LAI-Fook's experiment, which in turn can be compared directly with their continuum counterparts in Eqs. (1 \& 3) derived from hypo-elasticity.

\subsubsection{Kinematic Constraint}

In order for the distorted side pentagons to remain planar, which is a packing constraint imposed by the neighboring alveoli, and is in agreement with the assumption stated on pg. 11, Budiansky \& Kimmel [4] derived a scalar valued planar constraint that can be expressed in terms of the displacements $y$ as

$$
\xi=g \cdot \mathbb{y}+\frac{1}{2} \mathbb{y} \cdot \boldsymbol{h} \mathbb{y},
$$

wherein vector $g$ has components

$$
g=\frac{\sec (\omega)}{2}\left\{\begin{array}{c}
1 \\
\phi \\
-(1+2 \phi) \\
-1
\end{array}\right\}
$$

and matrix $\boldsymbol{h}$ has symmetric components

$$
\boldsymbol{h}=\left[\begin{array}{cccc}
0 & 0 & 0 & \phi-1 \\
0 & 0 & 2+2 \phi & 0 \\
0 & 2+2 \phi & 0 & -(1+3 \phi) \\
\phi-1 & 0 & -(1+3 \phi) & 0
\end{array}\right]
$$

Equations (32-34) follow from both their's and our's assumptions regarding how the side 
pentagons distort, which are different assumptions. In our case, they arise from equating the rise-over-run in the trapezoidal and triangular height expressions found in Eq. (104).

\subsubsection{Variational Analysis}

Following Budiansky \& Kimmel $[4,36]$, the principle of virtual work is applied to the constant pressure $P_{\Omega}$ experiment of LAI FooK et al. [40], cf. Fig. 1 and its protocol on pg. 2, to produce the following variational formulation.

A Lagrange multiplier $\lambda P_{\Omega} V_{\Omega}$ (where $\lambda$ is distinct from LAMÉ's constant $\lambda$, and $P_{\Omega} V_{\Omega}$ normalizes its effect) is introduced so that the variational form of the constraint equation (32) becomes

$$
\lambda P_{\Omega} V_{\Omega} \delta \xi=\lambda P_{\Omega} V_{\Omega}(g+\boldsymbol{h} \not) \cdot \delta y .
$$

The variational form of Eq. (9), but applied to the BVP of interest here, therefore becomes

$$
\begin{aligned}
10\left(F_{A B} \delta L_{A B}+F_{B C} \delta L_{B C}+F_{C D} \delta L_{C D}\right) \\
\quad+2 T_{R} \delta A_{R}+10 T_{D} \delta A_{D} \\
+\lambda P_{\Omega} V_{\Omega} \delta \xi=P_{\Omega} \delta V+\delta \pi .
\end{aligned}
$$

When the series expansions of Eqs. (21, 22, 24, $26 \& 32$ ) are substituted into Eq. (36), one gets

$$
\begin{aligned}
& 5 L_{\Omega}^{2}\left(\frac{F_{A B}}{L_{A B}}\left(A_{A B}+A_{A B} \mathbb{Y}\right)\right. \\
& +\frac{F_{B C}}{L_{B C}}\left(A_{B C}+A_{B C} \mathbb{V}\right) \\
& \left.+\frac{F_{C D}}{L_{C D}}\left(A_{C D}+A_{C D} \mathbb{y}\right)\right) \cdot \delta \mathbb{y} \\
& +2 A_{\Omega}\left(T_{R}\left(\mathbb{B}_{R}+\boldsymbol{B}_{R} \mathbb{Y}\right)\right. \\
& \left.+5 T_{D}\left(\mathbb{B}_{D}+\boldsymbol{B}_{D} \mathbb{y}\right)\right) \cdot \delta \mathbb{y} \\
& +\lambda P_{\Omega} V_{\Omega}(g+\boldsymbol{h} \Downarrow) \cdot \delta \Downarrow \\
& =P_{\Omega} V_{\Omega}\left(\mathbb{C}+C_{\mathbb{V}}\right) \cdot \delta_{\mathbb{y}} \\
& +\sigma V_{\Omega} \mathbb{f} \cdot \delta y,
\end{aligned}
$$

which must remain satisfied for every $\delta y$.
To determine the local elastic properties $\tilde{\mu}$ and $\tilde{v}$ of such a continuum, one seeks an incremental solution to the above variational problem for loading $\mathrm{d} \sigma$, which in turn causes an incremental displacement $\mathrm{d} y$, with constraint $\mathrm{d} \lambda$. This solution is valid within a small neighborhood around the reference state $\left(P_{\Omega}, L_{\Omega}\right)$, and it takes on a finite-element like form of

$$
\begin{aligned}
\boldsymbol{K} \cdot \mathrm{d} y+g \mathrm{~d} \lambda & =f \frac{\mathrm{d} \sigma}{P_{\Omega}}, \\
g \cdot \mathrm{d} y & =0
\end{aligned}
$$

where $\boldsymbol{K}=\boldsymbol{K}_{1 D}+\boldsymbol{K}_{2 D}+\boldsymbol{K}_{3 D}$ is the structure's stiffness matrix. Equation (38) consists of five equations in five unknowns: $\mathrm{d} y_{1}, \mathrm{~d} y_{2}, \mathrm{~d} y_{3}$, $\mathrm{d} y_{4}$, and $\mathrm{d} \lambda$.

To construct the stiffness matrix $\boldsymbol{K}_{1 D}$ that associates with the one-dimensional truss elements, one computes the gradients of their associated terms in Eq. (37), which generalize to a formula like

$$
\begin{aligned}
& \frac{\mathrm{d}}{\mathrm{d} \mathbb{y}}\left(\frac{F}{L}(A+\boldsymbol{A} \mathbb{y})\right) \cdot \mathrm{d} \mathbb{y} \\
& =\left\{\frac{F}{L} \boldsymbol{A}+\frac{1}{2}\left(\frac{\mathrm{d} F}{\mathrm{~d} L}-\frac{F}{L}\right)\right. \\
& \left.\times \frac{(A+\boldsymbol{A} \mathbb{y}) \otimes(A+\boldsymbol{A} \mathbb{y})}{1+A \cdot y+\frac{1}{2} \mathbb{y} \cdot \boldsymbol{A} y}\right\} \cdot \mathrm{d} \mathbb{y} .
\end{aligned}
$$

Letting $\sigma \rightarrow 0$, so that $L \rightarrow L_{\Omega}$ and $\boldsymbol{y} \rightarrow \mathbb{D}$, one arrives at the desired tangent at $\left(P_{\Omega}, L_{\Omega}\right.$

$$
\begin{aligned}
& \left.\frac{\mathrm{d}}{\mathrm{d} y}\left(\frac{F}{L}(A+\boldsymbol{A} y)\right)\right|_{\Omega} \cdot \mathrm{d} y=\left\{\left.\frac{F}{L}\right|_{\Omega} \boldsymbol{A}\right. \\
& \left.+\left.\frac{1}{2}\left(\frac{\mathrm{d} F}{\mathrm{~d} L}-\frac{F}{L}\right)\right|_{\Omega} A \otimes A\right\} \cdot \mathrm{d} y \text {. }
\end{aligned}
$$

Pulling everything together, where it is noted that $F_{A B} \rightarrow F_{\Omega}$ and $\mathrm{d} F_{A B} /\left(\mathrm{d} L_{A B} / L_{A B}\right) \rightarrow$ $\mathrm{d} F_{\Omega} /\left(\mathrm{d} L_{\Omega} / L_{\Omega}\right)$ as $\sigma \rightarrow 0$, etc., leads to the 
following stiffness matrix for the line elements

$$
\begin{aligned}
\boldsymbol{K}_{1 D} & =\frac{8 \cos ^{2}(\omega)}{P_{\Omega} L_{\Omega}^{2} \phi^{3}}\left\{F_{\Omega}\left(A_{A B}+A_{B C}+A_{C D}\right)\right. \\
+ & \frac{1}{2}\left(\left.\frac{\mathrm{d} F}{\mathrm{~d} L / L}\right|_{\Omega}-F_{\Omega}\right)\left(A_{A B} \otimes A_{A B}\right. \\
& \left.\left.+A_{B C} \otimes A_{B C}+A_{C D} \otimes A_{C D}\right)\right\}
\end{aligned}
$$

Following the same line of attack, one arrives at a stiffness matrix for the two-dimensional surface elements of a dodecahedron, it being

$$
\begin{aligned}
\boldsymbol{K}_{2 D} & =\frac{2 \cos (\omega)}{P_{\Omega} L_{\Omega} \phi^{2}}\left\{T_{\Omega}\left(\boldsymbol{B}_{R}+5 \boldsymbol{B}_{D}\right)\right. \\
+ & \left.\left.\frac{\mathrm{d} T}{\mathrm{~d} A / A}\right|_{\Omega}\left(\mathbb{B}_{R} \otimes \mathbb{B}_{R}+5 \mathbb{B}_{D} \otimes \mathbb{B}_{D}\right)\right\} .
\end{aligned}
$$

Likewise, the stiffness matrix governing the three-dimensional volume response is simply

$$
K_{3 D}=-C .
$$

Quantities for $F$ and $T$, and there derivatives $\mathrm{d} F /(\mathrm{d} L / L)$ and $\mathrm{d} T /(\mathrm{d} A / A)$, are completely general in this construction. They are to be supplied by external constitutive expressions.

Because $\lambda \rightarrow 0$ and $\boldsymbol{y} \rightarrow \mathbb{Q}$ as $\sigma \rightarrow 0$, the constraint equation (35) reduces to the second formula in Eq. (38); consequently, matrix $\boldsymbol{h}$ does not impact the final variation formulation.

\section{Fibers}

With only a few exceptions, e.g., in [35] where the alveolar faces are modeled as membranes, or in [70] where alveolar ducts are modeled as hexagonal arrays of springs, most existing geometric models for the alveolar microstructure of lung are constructed as pinned space trusses, whose bar elements are modeled as fibers loaded in tension. There are, however, substantial differences between the force/extension laws that are used, viz., the constitutive responses assigned to describe the behavior of the linkages that make up these space trusses. Here we review those that have been used, others that could be used, and those advocated for use.

In terms of the dodecahedral structural model discussed in $\S 3$, it is useful to express alveolar fiber models in terms of the forces $F$ they carry, and their local tangent moduli $L \mathrm{~d} F / \mathrm{d} L=\mathrm{d} F / \mathrm{d} \ln L$, both of which are taken to be functions of septal length $L$. In all of the models discussed below, except KimMEL's model, the fiber's elastic modulus $E$ is taken to be the tangent modulus pertaining to the initial state associated with zero pressure $P=0$ and gage length $L_{0}$. Several of these models require a second material parameter, which is denoted as $B$ throughout, but whose physical interpretations vary from model to model. The Eulerian (or true) stress carried by a fiber is quantified by $\sigma=\lambda S$, where $\lambda=L / L_{0}$ is the stretch and $S=F / A_{0}$ is the Lagrangian (or engineering) stress, with $A_{0}$ being the initial cross-sectional area of the fiber.

\subsection{Assumptions}

The following assumptions are considered.

- Each fiber in a truss element assumes the behavior of an incompressible cylinder described by its associated material type.

- The force carried by each fiber is shared equally between the three neighboring alveoli to which it belongs.

- Elastin is modeled via the hyper-elastic neo-Hookean solid [1, 67].

- Collagen is modeled via the hypo-elastic FunG-like solid [16].

- The elastin and collagen fiber networks act independently $[44,56]$, their elements are 
loaded in parallel [8], and their physical dimensions are the same $[47,56]$.

\subsubsection{First Assumption}

Although lung is a highly compressible material, the constituents that provide its structural integrity are, to a good approximation, incompressible. What this really means is that the ratio of their shear-to-bulk moduli is smaller than about one part in a hundred, which is common amongst soft solids. This being the case, structural truss elements comprising a dodecahedral model ought to be taken to be incompressible materials whose governing equations depend upon the material that each member is made from, and the BVP that is imposed upon it.

\subsubsection{Second Assumption}

This follows from geometric patterning considerations.

\subsubsection{Third Assumption}

Elastin is a protein that is an isotropic polypeptide elastomer built from monomeric units that have between three and six peptides in each repeating unit, with a total of about 100 monomers in a typical elastomer chain, plus elastin is entropic above about $25^{\circ} \mathrm{C}$ [67]. Therefore, its physiochemical properties are compatible with those used to derive the classical theory of rubber elasticity from statistical mechanics $[1,63]$.

The load bearing elastin fibers are located predominantly along the alveolar septa [47], i.e., along the pentagonal edges of the dodecahedron. As these are taken to be pinned truss elements loaded under tension only, it follows that the elastin fibers in this microstructural model are described by uniaxially stretched neo-Hookean rods so that $S=\mu\left(\lambda-\lambda^{-2}\right)$ [63, Eq. 5.3]. (Equations $52 \& 53$ quantify its force and tangent modulus.) The elastic (Young's) modulus $E=3 \mu$ of single, unswollen, elastin fibers (5-8 $\mu \mathrm{m}$ diameter) extracted from purified, bovine, ligamentum nuchae has a value of $1.18 \pm 0.03 \mathrm{MPa}$ at $37^{\circ} \mathrm{C}$ (glassy modulus) [1] When osmotically swollen, its elastic modulus can diminish to $0.17 \pm 0.01 \mathrm{MPa}$ (rubbery modulus) [42]. This is a large span of admissible values. The neo-Hookean model describes the experimental data for elastin to about $\lambda=2$, which exceeds the expected stretch along a septal line in a typical alveolus.

The hypo-elastic Fung model has also been fit to AARON \& GosLINE's [1] fiber data yielding parameters of $\mu=210 \pm 48 \mathrm{kPa}$ and $\beta=$ $3.06 \pm 0.38$ [16]. The model describes all of their data up to fiber rupture, which is around $\lambda \approx 2.5$, with a goodness of fit of $R^{2}=0.976$.

Matsuda, Fung \& Sobin [44] measured the diameter of elastin fiber bundles at the alveolar mouths in human lung at $7.11 \pm 2.93 \mu \mathrm{m}$, with the reported error being plus/minus one standard deviation from a sample size of 450 taken from a single lung. Sobin, Fung \& Tremer [56] measured the elastin fiber diameter at $D_{e}=$ $1.106 \pm 0.552 \mu \mathrm{m}$ in the inter-alveolar septa from 2030 samples taken from 5 human lungs.

From histological measurements, OLDMIXON \& Hoppin [47] determined that four-fifths of all parenchymal elastin resides at the cable locations comprising an alveolar unit cell, which associate with the truss elements in the dodecahedral model, while the remaining one-fifth of the elastin belongs to the alveolar walls and septal triple points. They measured the volume density (fiber volume to alveolar volume) to be $255 \pm 28 \times 10^{-6}$ in canine lung.

\subsubsection{Fourth Assumption}

Much of the author's (ADF) research efforts over the past five years has focused around the observation that the elastic behavior of collagenous materials is well described by the hypo- 
elastic representation of FunG's law [16, 21]. Collagen, like elastin, contributes to the transmission of tractions along the truss elements in the dodecahedron, and as such, is modeled via stretchable uniaxial rods with a material response of $\lambda^{2} \mathrm{~d} S / \mathrm{d} \lambda=E+B \lambda S$ [16]. (Equations $56 \& 57$ describe its fiber force and tangent modulus.)

Matsuda, Fung \& Sobin [44] measured the diameter of collagen fiber bundles at alveolar mouths in human lung at $4.98 \pm 2.24 \mu \mathrm{m}$, with the reported error being plus/minus one standard deviation from a sample size of 3,095 taken from a single lung. SobIn, Fung \& TREMER [56] measured the collagen fiber diameter at $D_{c}=1.053 \pm 0.539 \mu \mathrm{m}$ in the inter-alveolar septa of 6,902 samples taken from 14 human lungs. Oldmixon \& Hoppin [47] measured the volume density of collagen fibers in the cabling locations of alveoli to be $252 \pm$ $38 \times 10^{-6}$ for canine lung.

Collagen and elastin fiber dimensions are observed to be approximately equal in the truss regions of alveolar geometry. This has been shown to be true in both the diameter measurements of SobIN et al. [56] and the volume-fraction measurements of OLDMIXon \& Hoppin [47]. If one considers the fiber diameters for both collagen and elastin to be $1 \mu \mathrm{m}$, as measured by Sobin et al. in humans, and if one considers the volume fraction of fiber per unit dodecahedral cell to be $255 \times 10^{-6}$, as measured by OLDMIXON $\&$ HoppIN in canines, then one arrives at a reference pentagonal length of $L_{0}=63 \mu \mathrm{m}$, and a nominal, alveolar, cell diameter of $\bar{D}_{0}=$ $155 \mu \mathrm{m}$ at zero transpulmonary pressure. This result is consistent with the nominal diameters measured by SoBIN et al. in humans, as reported on pg. 8 and visualized in Fig. 10.

The geometry of our alveolar model is therefore established; it being summarized in Table 1, where published dimensions from similar models are also tabulated. Data from both dodecahedron and truncated octahedron models

\begin{tabular}{|c|c|c|c|}
\hline Reference & $D_{f}(\mu \mathrm{m})$ & $L(\mu \mathrm{m})$ & $\bar{D}(\mu \mathrm{m})$ \\
\hline here & $1 / 1$ & 63 & 170 \\
{$[9]$} & $3.35 / 2.45$ & 45 & - \\
{$[39]$} & 2 & 60 & 185 \\
\hline
\end{tabular}

Table 1: Basic geometric dimensions used to model lung alveoli. Dimensions correspond to zero transpulmonary pressure. $D_{f}$ is the fiber diameter. When two diameters are given, the first associates with collagen and the second with elastin. $L$ is the septal length or distance between septal junctions. $\bar{D}$ is the nominal diameter of an alveolar unit cell. A dash implies that its value was not reported.

are presented, which accounts for what might otherwise be considered to be disparities between these data.

\subsubsection{Fifth Assumption}

The extensive histological studies of MAtsudA et al. [44], Oldmixon \& Hoppin [47], and of Sobin et al. [56] provide no discussion of, nor evidence for suggesting that collagen and elastin parenchymal fibers are either mechanically or chemically interconnected in any significant way. This suggests that these two fiber types, in a mechanical sense, act in parallel with one another. This observation has also been used by others to simplify their mechanical models of lung, e.g., Denny \& Schroter [9],

There are, however, examples in biology where collagen and elastin have a substantial mechanical connection, e.g., the ligamentum propatagiale that supports the leading edge of skin along avian wings [3]. In these struts, a central elastin fiber transitions into collagen ligaments at its two ends. One ligament attaches to the humerus, and the other attaches to the radius and ulna. Here Nature has designed elastin and collagen fibers to be loaded in series; whereas, Nature has designed the elastin and collagen fibers in lung parenchyma to carry 
their loads in parallel.

\subsection{Models}

Here we review material models that have been used in alveolar truss models, those that we advocate using, and others that could potentially be considered.

\subsubsection{Hooke's Law}

This well-known elastic law says $\sigma=E \epsilon$, with $\sigma=F / A_{0}$ and $\epsilon=\left(L-L_{0}\right) / L_{0}$ being the engineering stress and strain that arise from the classical theory of linear elasticity. This model predicts a fiber force of

$$
F=E A_{0} \frac{L-L_{0}}{L},
$$

whose tangent modulus is

$$
\frac{\mathrm{d} F}{\mathrm{~d} L / L}=E A_{0}-F
$$

that in the limit as $L \rightarrow L_{0}$ becomes $E A_{0}$, or $L \mathrm{~d} S /\left.\mathrm{d} L\right|_{L=L_{0}}=E$, as expected.

\subsubsection{Carton's Model}

In the 1962 paper of CARTON et al. [5], they propose a material model for elastin filaments 9 where $\epsilon=\epsilon_{\max }\left(1-\mathrm{e}^{-S / E \epsilon_{\max }}\right)$ (in our notation) that, when cause and effect are switched around, becomes ${ }^{10}$

$$
F=-E A_{0} \frac{L_{\max }-L_{0}}{L_{0}} \ln \left(\frac{L_{\max }-L}{L_{\max }-L_{0}}\right),
$$

whose tangent modulus is

$$
\frac{\mathrm{d} F}{\mathrm{~d} L / L}=E A_{0} \frac{L}{L_{0}} \frac{L_{\max }-L_{0}}{L_{\max }-L},
$$

where $L_{\max }$ is the maximum, septal, fiber length, i.e., it's length at fiber rupture. This is a model in two parameters: $E$ and $L_{\max } / L_{0}$.

\subsubsection{Fung's Law}

In 1967 FuNG [22] proposed a model very similar to the one of CARTON et al. [5], but with the dependent and independent variables being swapped; specifically, Fung proposed a model, a.k.a. Fung's law $\mathrm{d} S / \mathrm{d} \lambda=E+B S$, that when integrated produces the fiber response

$$
F=\frac{E A_{0}}{B}\left(\mathrm{e}^{B \epsilon}-1\right),
$$

whose tangent modulus is

$$
\frac{\mathrm{d} F}{\mathrm{~d} L / L}=\frac{L}{L_{0}}\left(E A_{0}+B F\right)
$$

where $B$ and $E$ are the material parameters. In Fung's paper, and in the authors' implementations of his law, e.g., [18], $B$ is denoted as $\beta$.

\subsubsection{Kimmel's Model}

In the papers of Kimmel et al. [4, 36, 37], the tangent modulus for fiber stress is taken to be

$$
\frac{\mathrm{d} F}{F}=B \frac{\mathrm{d} L}{L} \quad \text { or } \quad \frac{\mathrm{d} F}{\mathrm{~d} L / L}=B F,
$$

which integrates to

$$
F=\left(\frac{L}{L_{0}}\right)^{B}-1
$$

This model has a single material parameter, viz., $B$, that relates the logarithmic rates of traction $F$ to length $L$. Because the tangent modulus becomes zero in the reference state, this model cannot permit $F$ to become zero; therefore, it necessarily imposes a constraint of $F>0$.

9. CARTon et al. say their model is for elastin, but it actually describes the behavior of collagen.

10. The paper of DaLE et al. [6] rewrites the model of CARTON et al. [5] in terms of Eulerian variables for stress and strain; whereas, Lagrangian descriptions are used here. Its Eulerian representation is used in the finite element model of Kowe et al. [39] and in all of the models of Denny \& Schroter $[7,8,9,10]$. 
The units in this integrated form do not make physical sense. This model lacks a modulus.

\subsubsection{Neo-Hookean Solid}

Except for the Hookean solid, which follows from a more general 3D theory, viz., linear elasticity, the CarTon, Fung, and Kimmel fiber models are all 1D empirical relationships. Like the Hookean model, the remaining fiber models are mathematical consequences arising from more general 3D theories that solve a 1D BVP describing the extension of an incompressible rod.

The first such model to be considered is the neo-Hookean solid [63] that, in simple extension, has a force/stretch relationship of

$$
F=\frac{E A_{0}}{3}\left(\frac{L}{L_{0}}-\frac{L_{0}^{2}}{L^{2}}\right),
$$

whose tangent modulus is

$$
\frac{\mathrm{d} F}{\mathrm{~d} L / L}=E A_{0} \frac{L_{0}^{2}}{L^{2}}+F,
$$

which has one material parameter: $E$.

\subsubsection{Mooney-Rivlin Solid}

A generalization of the neo-Hookean solid is the so-called Mooney-RivLin solid [63], whose governing relation for the $1 \mathrm{D}$ extension of a rod leads to a force response of

$$
\begin{aligned}
F=\frac{E A_{0}}{3}[(1-B) & \left(\frac{L}{L_{0}}-\frac{L_{0}^{2}}{L^{2}}\right) \\
+ & \left.B\left(1-\frac{L_{0}^{3}}{L^{3}}\right)\right]
\end{aligned}
$$

whose tangent modulus is

$$
\begin{aligned}
& \frac{\mathrm{d} F}{\mathrm{~d} L / L} \\
& =\frac{E A_{0}}{3}\left[(1-B)\left(\frac{L_{0}}{L}+2 \frac{L_{0}^{2}}{L^{2}}\right)\right. \\
& \left.+3 B \frac{L_{0}^{3}}{L^{3}}\right],
\end{aligned}
$$

which has two parameters: $E$ and $B$, where $E$ is the elastic modulus at zero force, and $B$ partitions the response between two, competing, strain measures. In the literature, these constants are denoted via $C_{1}=E(1-B) / 3$ and $C_{2}=E B / 3$, which are Mooney's [46] coefficients.

\subsubsection{Hypo-Fung Solid}

This material response is a derived consequence from a three-dimensional theory $[15,17]$ based upon FunG's empirical law, as presented in \$4.2.3. The theory's prediction for simple extension [16] is a power-law, instead of the exponential that arises from FunG's empirical law; specifically,

$$
F=\frac{E A_{0}}{B+1}\left(\frac{L^{B}}{L_{0}^{B}}-\frac{L_{0}}{L}\right),
$$

whose tangent modulus is

$$
\frac{\mathrm{d} F}{\mathrm{~d} L / L}=E A_{0} \frac{L_{0}}{L}+B F,
$$

which is a model in two parameters: $E$ and $B$.

The difference between formulæ ( $49 \& 57$ ) has to do with how the independent variable $L$ enters into the right-hand side. This subtlety has a strong effect on their integrated forms, as we shall soon see.

Model (56) reduces to the hypo-Hookean solid whenever $B=1$, which is Truesdell's [65] theory for an incompressible hypo-elastic solid of grade zero. 


\subsection{Model Comparison}

To gain insight into how these various material models behave, their stretch $\lambda=L / L_{0}$ vs. force $F$ responses are plotted in Fig. 12, and their force $F$ vs. tangent modulus $\mathrm{d} F /(\mathrm{d} L / L)$ responses are plotted in Fig. 13. ${ }^{11}$

These models fall into two groups: the CARTON, Fung, and hypo-Fung models are reasonable formulæ for describing collagen, while the Hookean, neo-Hookean, hypo-Hookean, and Mooney-RivLIN models are reasonable formulæ for describing elastin. Strictly speaking, the Hookean model should be removed from consideration, because it is derived from a theory that is applicable only for infinitesimal strains, which is not the case here. Likewise, the Fung model should give way to the hypo-Fung model, as the latter follows from a general theory, while the former does not. Furthermore, the hypo-Hookean model is a special case of the hypo-Fung model, viz., $B=1$.

Parametric values that approximate their respective responses for collagen or elastin have been assigned, with physical dimensions being assigned according to realistic dimensions in mammalian alveoli. The collagen responses were artificially set to coincide with the elastic response at a stretch of $\lambda \approx 1.5$ for comparative purposes. There is a lot more flexibility in the hypo-Fung model than there is in the CARTON model at fitting a shape.

\section{Membranes}

Rods are used to model the truss-work within a mechanical model for an alveolus, while membranes are reasonable candidates for representing the alveolar walls. There are twelve membrane panels that comprise a dodecahedron.

\subsection{Assumptions}

In addition to the modeling assumptions that apply to the truss elements, others are needed for the panel elements.

- Each alveolar face is modeled as an incompressible membrane.

- The surface traction carried by each membrane face is shared by the two neighboring alveoli to which it belongs.

- The surface tension carried by the surfactant film belongs wholly to the alveolus in which it resides; it is not shared.

- Surface tension $\gamma$ caused by surfactant has the response of a uniformly stretched, neo-Hookean membrane whose elastic modulus varies with concentration $\Gamma$.

- The concentration of surfactant $\Gamma$ is governed by three formulæ: the first describes its liquid expansion, the second describes its liquid condensation, and the third describes its solid-like regime [50].

\subsubsection{First Assumption}

The arguments that support this assumption for panel elements are the same as those that support the corresponding assumption for truss elements.

\subsubsection{Second and Third Assumptions}

These follow from geometric patterning considerations.

\subsubsection{Fourth Assumption}

We can strengthen our case for this assumption by considering that the surfactant mono-layer is 11. The model of KimmeL is not shown in these figures because it has no means to assign an elastic modulus. 


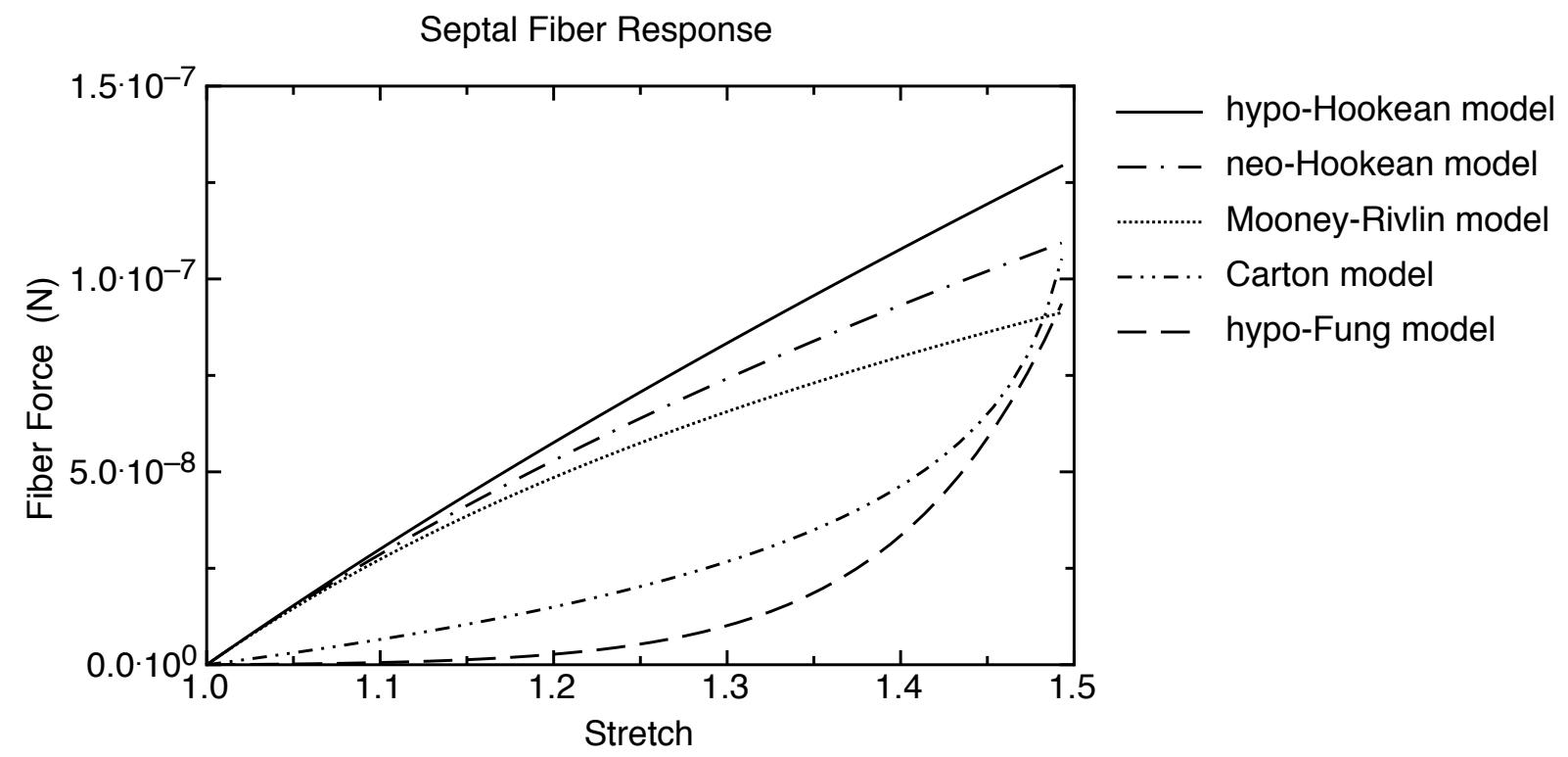

Figure 12: Illustrative $\lambda=L / L_{0}$ vs. $F$ plots for the septal fiber response predicted by material models $(46,52,54 \& 56)$ with the geometric parameters set at: $L_{0}=63 \mu \mathrm{m}$, $L_{\max }=95 \mu \mathrm{m}, D_{0}=1 \mu \mathrm{m}$. The elatic modulus $E$ was set at: $E=1.2 \mathrm{MPa}$ for the hypoHookean, neo-Hookean, and Mooney-Rivlin solids; $E=225 \mathrm{kPa}$ for the Carton solid; and $E=10 \mathrm{kPa}$ for the hypo-Fung solid. The dimensionless parameter $B$ was set at: $B=0.5$ for the Mooney-Rivin solid, and $B=16$ for the hypo-Fung solid.

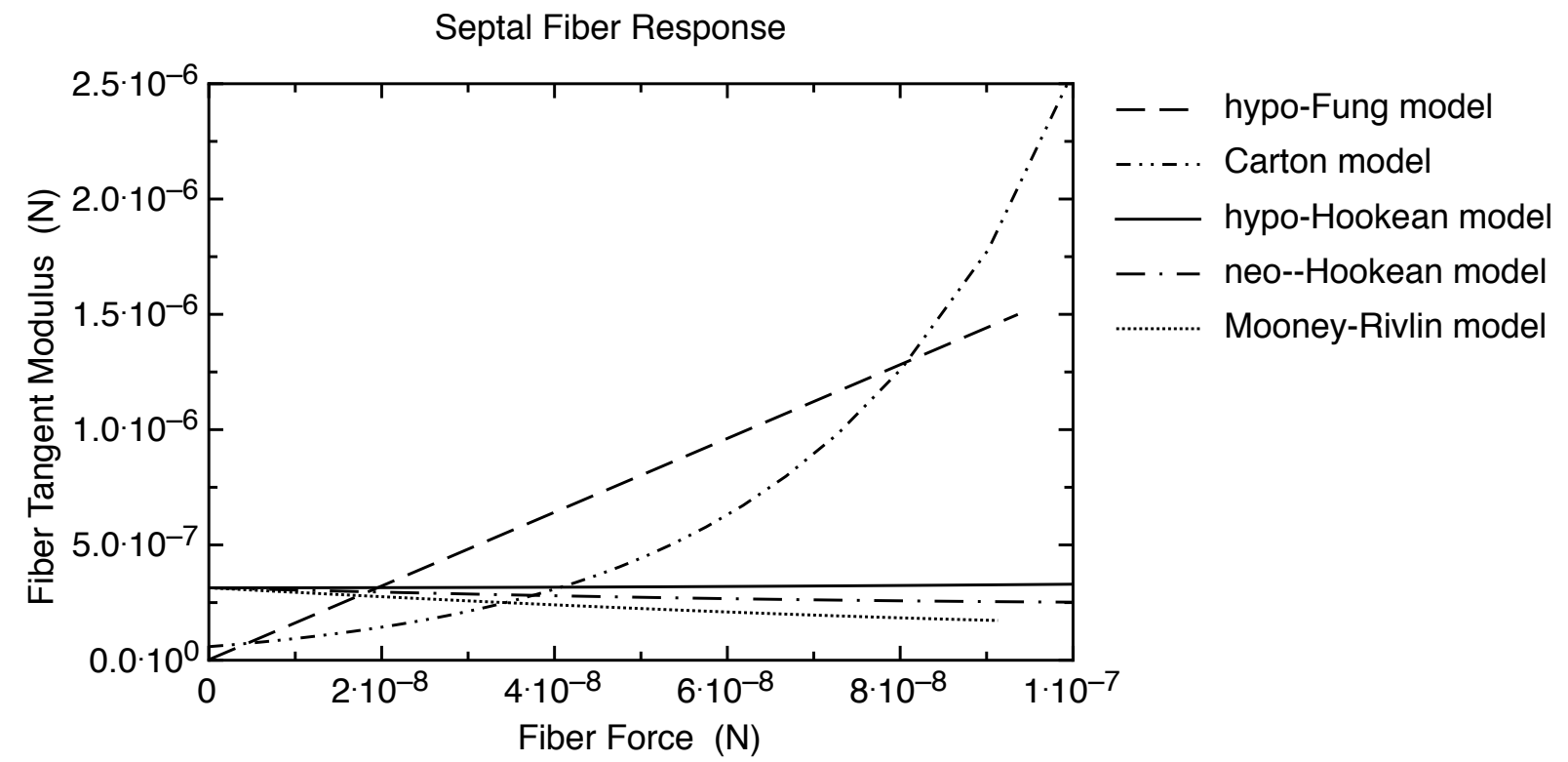

Figure 13: Illustrative $F$ vs. $\mathrm{d} F /(\mathrm{d} L / L)$ plots, i.e., Fung plots, for the septal fiber response predicted by material models $(47,53,55 \& 57)$ with the geometric parameters set at: $L_{0}=$ $63 \mu \mathrm{m}, L_{\max }=95 \mu \mathrm{m}, D_{0}=1 \mu \mathrm{m}$. The elatic modulus $E$ was set at: $E=1.2 \mathrm{MPa}$ for the hypo-Hookean, neo-Hookean, and Mooney-Rivlin solids; $E=225 \mathrm{kPa}$ for the Carton solid; and $E=10 \mathrm{kPa}$ for the hypo-Fung solid. The dimensionless parameter $B$ was set at: $B=0.5$ for the Mooney-Rivlin solid, and $B=16$ for the hypo-Fung solid. 
a rubberlike material made up of a variety of macromolecular complexes held in suspension along the surface layer. These complexes are comprised of about $95 \%$ lipids and $5 \%$ proteins [62]. The disaturated phospholipid dipalmitoylphosphatidylcholine makes up about half of the lipid content, and is primarily responsible for the surface tension reducing property of the surfactant [54].

Because the importance of surfactant to the mechanics of lung is due to its surface tension properties, not its bulk fluidic properties, one can consider the 'skin' of this fluid as being a rubberlike membrane, and ignore the affect of the bulk fluid that resides underneath.

\subsubsection{Fifth Assumption}

The surface tension carried by a mono-layer of surfactant at the air/liquid lining along alveolar walls is a complex response governed by physio-chemical processes through which the force is modulated [54, 62]. OTIs et al. [50] decomposed the kinetics governing the interfacial surfactant concentration $\Gamma$ into three regimes.

In the first region (the liquid-expanded regime) the concentration of surfactant at the liquid/air interface is bounded from above by $\Gamma<\Gamma^{\star}$, with $\Gamma^{\star}$ being the maximum equilibrium concentration of surfactant at the interface, i.e., within the surface experiencing tension. In this regime surfactant migrates between the bulk fluid and the liquid/air interface that contains it. Here OTIs et al. assume that LANGMUIR kinetics control the transport of surfactant, as described by

$$
\begin{aligned}
\frac{\mathrm{d}(\Gamma A)}{\mathrm{d} t} & =A\left(k_{1} C\left(\Gamma^{\star}-\Gamma\right)-k_{2} \Gamma\right), \\
& =c_{1} \Gamma^{\star} A-c_{2} \Gamma A,
\end{aligned}
$$

where $k_{1}$ and $k_{2}$ are the LANGMUIR adsorption (bulk $\rightarrow$ surface) and desorption (surface $\rightarrow$ bulk) diffusion coefficients, with $c_{1}=k_{1} C$ and $c_{2}=k_{1} C+k_{2}$. Parameter $C$ represents the concentration of surfactant in the fluid.

In the second region (the liquid-condensed regime) surfactatant concentration at the liquid/ air interface varies between $\Gamma^{\star} \leq \Gamma \leq \Gamma_{\max }$. Here the surface layer is taken to be insoluble, i.e., there is no exchange in surfactant between it and the bulk fluid below it. OTIs et al. consider that the concentration of surfactant can only change in this domain if the area changes, so in this regime

$$
\Gamma=\Gamma^{\star} \frac{A^{\star}}{A},
$$

up to a value of $\Gamma=\Gamma_{\max }$ that denotes the maximum dynamic concentration of surfactant that can exist at the liquid/air interface.

In the third and final region (the solid-like regime) the concentration of surfactant within the air/liquid interface is saturated at

$$
\Gamma=\Gamma_{\max } .
$$

Here the surface tension $\gamma$ is at its minimum. The lipid mono-layer pancakes up on itself in this region [62], much like the wind stacking up ice flows one atop another when the ice goes out on a large body of water.

Values reported in the literature for these constants are [9]: $c_{1}=1.168 \mathrm{~s}^{-1}$ and $c_{2}=$ $1.184 \mathrm{~s}^{-1}$, with $\gamma\left(\Gamma_{\max }\right)=2 \mathrm{dyn} / \mathrm{cm}$ and $\gamma\left(\Gamma^{\star}\right)=22.2 \mathrm{dyn} / \mathrm{cm}$.

\subsection{Models}

\subsubsection{Film}

LAPLACE's theory for surface tension $\gamma$ relates a pressure differential $\Delta p$ acting across a bubblelike surface to the principle radii of curvature $R_{1}$ and $R_{2}$ that describe that surface, cf. [14]; specifically,

$$
\Delta p=\gamma \frac{R_{1}+R_{2}}{R_{1} R_{2}} .
$$


Consequently, increasing surface tension $\gamma$ increases the bubble size at a fixed pressure differential across the surface, and vice versa.

A somewhat naïve adaptation of LAPLACE's physical law to an alveolus is presented below. Take one principle radius to associate with the nominal radius of an alveolar sac, and take the other principle radius to associate with the nominal radius of an alveolar mouth. If alveoli behave as thin films, then

$$
P_{M}=2 \gamma \frac{D+\bar{D}}{D \bar{D}}
$$

where $P_{M}$ is that fraction of the transpulmonary pressure $P$ which is carried by the surface of the membrane, $D$ is the nominal diameter of an alveolar mouth, and $\bar{D}$ is the nominal diameter of an alveolar sac.

As this characteristic pertains to the bulk response, it follows from Eq. (11) that

$$
P_{M} V=8 T A .
$$

Combining the two above equations leads to the relationship

$$
\begin{aligned}
T & =\frac{V}{4 A} \frac{D+\bar{D}}{D \bar{D}} \gamma(\Gamma(A)) \\
& \approx 1.138 \gamma(\Gamma(A)),
\end{aligned}
$$

with tangent modulus

$$
\frac{\mathrm{d} T}{\mathrm{~d} A / A}=\frac{V}{4 A} \frac{D+\bar{D}}{D \bar{D}} \frac{\mathrm{d} \gamma(\Gamma)}{\mathrm{d} \Gamma} \frac{\mathrm{d} \Gamma(A)}{\mathrm{d} A / A},
$$

where $T$ is the surface tension carried along a planar pentagonal surface in a dodecahedral model, while $\gamma$ is the surface tension carried along a thin film with curvature and a pore opening. The coefficient, whose value is approximately 1.138 , accounts for their geometric differences, viz., planar vs. curvalinear.

\subsubsection{Neo-Hookean Solid}

A neo-Hookean solid placed in plane-stress equi-biaxial (or isotropic) traction is described by a uniform state of stress $S=\mu\left(\lambda-\lambda^{-5}\right)$, which has units of force per unit area. The force per unit length along the surface carried by a membrane is therefore governed by $[63$, Eq. 5.7]

$$
T=\mu t_{0}\left(1-\frac{A_{0}^{3}}{A^{3}}\right),
$$

where $T$ is the planar surface tension, and $t_{0}$ is the initial thickness of the membrane. In the case of the alveolar wall, the shear modulus $\mu$ is taken to be a constant; however, in the case of the surfactant membrane, the shear modulus $\mu(A)$ is taken to be a function of surface area $A$. From self-similarity, $\lambda^{2}=D^{2} / D_{0}^{2}=A / A_{0}=$ $\Lambda$, where $D$ is the mean diameter of a septal face, while $\Lambda=A / A_{0}$ is its areal stretch. The relevant tangent modulus obeys

$$
\frac{\mathrm{d} T}{\mathrm{~d} A / A}=3\left(\mu t_{0}-T\right)+\frac{T}{\mu} \frac{\mathrm{d} \mu}{\mathrm{d} A / A} .
$$

In the case of surfactant, $\mu$ is given by the right-hand side of Eq. (64), while $\mathrm{d} \mu /(\mathrm{d} A / A)$ is given by the right-hand side of Eq. (65).

The differential equation (67) is very different from the ones that describe the collagen and elastin truss members, viz., Eqs. (53 \& 57). Equation (67) has a saturation state at $T=\mu t_{0}$, given that $\mathrm{d} \mu /(\mathrm{d} A / A)=0$ at $T=\mu t_{0}$, too, while the other two ODEs describe fiber behavior that continues to stiffen with deformation. This is a geometric effect, not a material effect; specifically, Eqs. (53 \& $67)$ both pertain to neo-Hookean solids. This is a desirable outcome, as it is an observed phenomenon of the surfactant film at TLC.

\subsubsection{Hypo-Hookean Solid}

There is one other material model whose solution for a uniformly stretched solid has a saturating value, i.e., an apparent ultimate strength; it is the hypo-Hookean solid, which produces a 
stress state of $S=3 \mu\left(\lambda-\lambda^{-1}\right)$ [18]. When written for a membrane, it becomes

$$
T=3 \mu t_{0}\left(1-\frac{A_{0}}{A}\right),
$$

whose tangent modulus is

$$
\frac{\mathrm{d} T}{\mathrm{~d} A / A}=3 \mu t_{0}-T+\frac{T}{\mu} \frac{\mathrm{d} \mu}{\mathrm{d} A / A},
$$

which saturates at traction $T=3 \mu t_{0}$, provided that $\mathrm{d} \mu /(\mathrm{d} A / A)=0$ at $T=3 \mu t_{0}$, too.

These two models saturate at different physical strengths. The neo-Hookean membrane saturates whenever the surface tension reaches the material's shear strength; whereas, the hypo-Hookean membrane saturates whenever the surface tension reaches the material's elastic strength. They also follow different $(T, \Lambda)$ trajectories to saturation. The neo-Hookean trajectory has a more abrupt transition into saturation than does the hypo-Hookean trajectory.

\section{Pressure-Volume Curves}

To demonstrate where we are headed, discussion is now presented on the heterogeneity of alveolar response within a single lung. The models discussed up to this point describe the response of a single alveolus. This model will be incorporated into a finite element package to allow full lung simulations. These simulations will require verification, just like our single alveolar models do. To this end, the following technology is being developed at PNNL by the authors (DRE, JPC, and REJ).

Global pressure-volume $(P V)$ curves are routinely acquired in both laboratory and clinical settings with a plethesmagraph. These curves represent an integrated response over the whole lung. However, lung ventilation, and therefore lung expansion, is markedly heterogeneous [53]. Local deviations from nominal

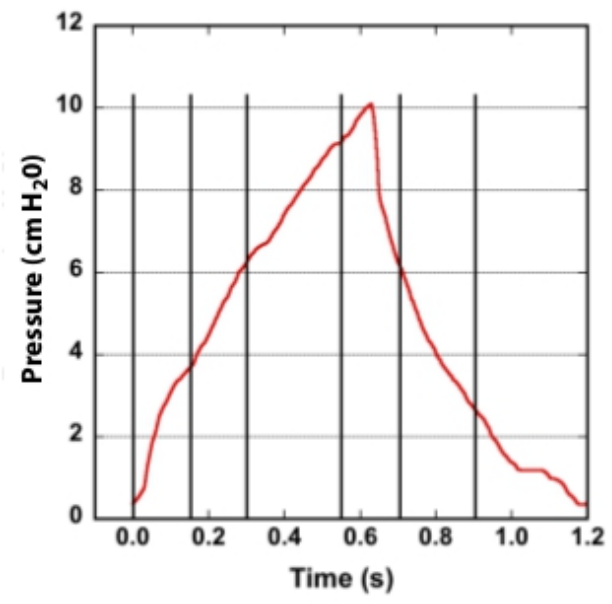

Figure 15: Pressure-time history of a typical breathing cycle subjected to a rat whose $P V$ response is displayed in Fig. 14 for one such loading cycle. CT images were acquired at 0, 150, 300, 550, 700, and $900 \mathrm{~ms}$.

heterogeneity have both clinical and pathological implications [30]. Spatially heterogeneous measurements of local $P V$ relationships are now possible at realistic breathing rates through a gated computed tomography.

A pressure $P$ volume-ratio $\mathrm{d} V / \mathrm{d} V_{0}$ relationship at two distinct points in the left and right lungs of a Sprague-Dawley rat is shown in Fig. 14. Below, we briefly describe the acquisition and processing of these data. It should be noted that the recorded pressure, shown in Fig. 15, is that of the ventilator. Nasal and local alveolar pressures will, in general, be different.

\subsection{Data Acquisition}

A $344 \mathrm{~g}$ male Sprague-Dawley rat was anesthetized with $3.5 \%$ isoflurane in oxygen and orally intubated with a 14 gauge catheter tube. The rat was then placed in the imaging tray of the CT scanner (eXplore 120, GE Healthcare) and connected to a computer-controlled me- 


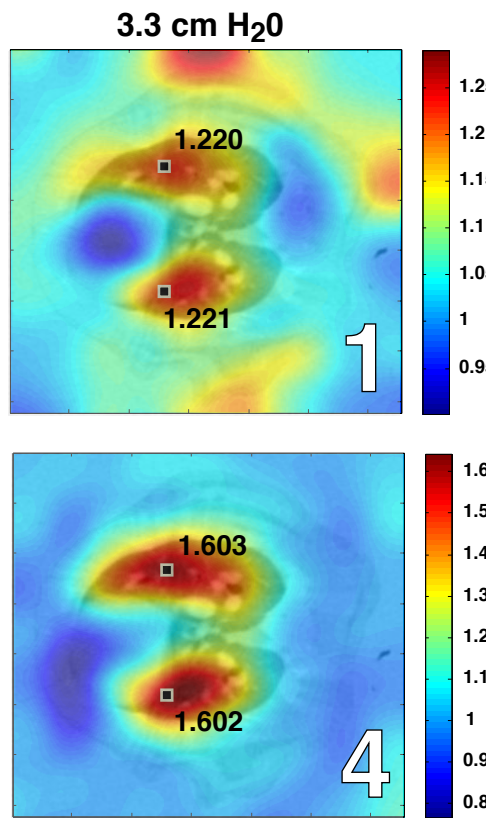

$5.8 \mathrm{~cm} \mathrm{H} \mathrm{H}_{2}$
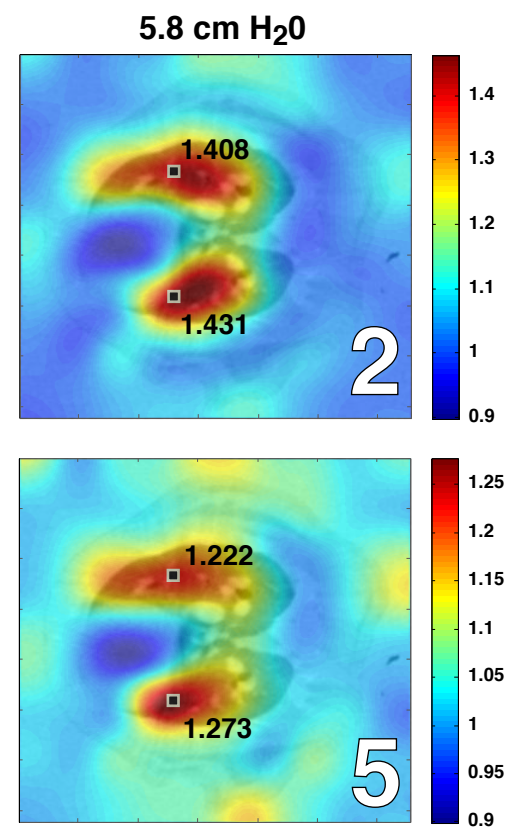

$2.2 \mathrm{~cm} \mathrm{H} \mathrm{O}$
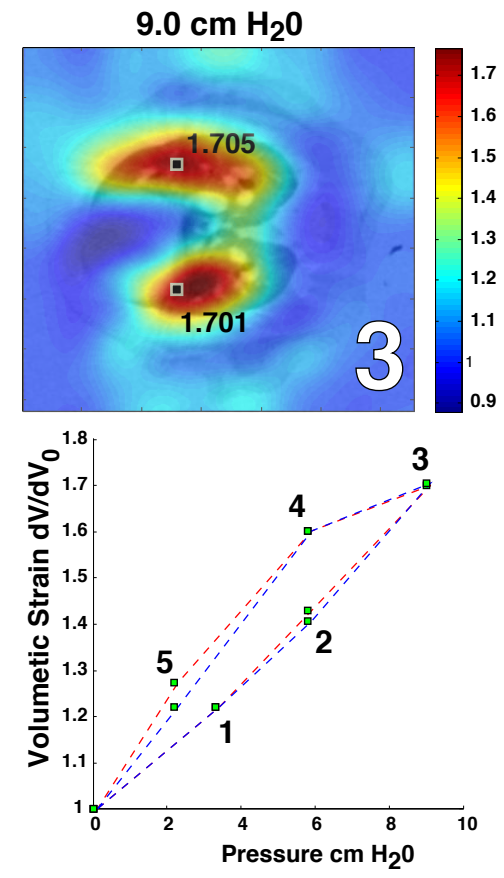

Figure 14: In vivo $P$ vs. $\mathrm{d} V / \mathrm{d} V_{0}$ curve for rat lung determined from a sequence of CT images acquired over a single breathing cycle. The top row of images relate to inflation. The bottom row of images relate to deflation. Each cycle begins and ends at the end of expiration.

chanical ventilator (SAR-830/AP, CWE Inc.) while being maintained on isoflurane. Ventilation parameters were: 50 breaths per minute, 1:1 inhale:exhale ratio, $\sim 10 \mathrm{~cm} \mathrm{H}_{2} \mathrm{O}$ peak inspiratory pressure, and $\sim 3.7 \mathrm{ml}$ tidal volume. A sigh was automatically incorporated every 30 breaths to a peak pressure of $24 \mathrm{~cm} \mathrm{H}_{2} \mathrm{O}$ and volume of $\sim 9.5 \mathrm{ml}$. The rat's body temperature was maintained at $37 \pm 1^{\circ} \mathrm{C}$ with warm water circulation.

A gating signal was sent to the CT scanner at the beginning of each inhalation cycle, except for each sigh and the three breaths immediately following. In order to capture the lungs at various points in the breathing cycle, the $\mathrm{CT}$ scanner was programmed with the following gating delays (in ms): 0, 150, 300, 550, 700, and 900 (see Fig. 15). The scanner acquired a single projection per breath and acquired all six gating delays (over 6 breaths) before incrementing to the next gantry angle. The CT imaging parameters were set at: $100 \mathrm{kVp}, 50 \mathrm{~mA}, 16 \mathrm{~ms}$ exposure time, 220 projections over over $190^{\circ}$, and a 50 micron isotropic resolution. Images were reconstructed to a 150 micron resolution to reduce noise and enhance airway contrast.

The short $16 \mathrm{~ms}$ exposure time allowed for projection acquisition during breathing with minimal blurring, obviating the need for intermediate breath holds and complicated breathing maneuvers. Total imaging time was approximately 45 minutes.

Following imaging, the rat was sacrificed with $\mathrm{CO}_{2}$ asphyxiation and prepared for lung airway casting, as described by PeRry et al. [51]. Silicone casting material, consisting of $10.5 \mathrm{~g}$ Dow Corning 734 flowable sealant, $2.5 \mathrm{~g}$ Dow Corning 200 fluid (20 CS), and $\sim 1 \mathrm{ml}$ Ultravist 300 (Bayer Healthcare) contrast agent, was thoroughly mixed and degassed under vacuum. Approximately $1.5 \mathrm{ml}$ of the mixture was injected into the lungs. After about 1 hour, the cast was CT imaged in situ with the following 
parameters: $100 \mathrm{kVp}, 50 \mathrm{~mA}, 20 \mathrm{~ms}$ exposure time, 720 projections over over $360^{\circ}, 2$ averages per projection angle, and a 50 micron isotropic resolution.

\subsection{Computation of the Displacement} Field

At different pressures, localized lung movement is semi-independent of the surrounding tissues such as the ribcage. To enable accurate calculations of deformations describing the changes in volumetric MRI data at different pressures, the impact of image information outside of the lung was effectively minimized. This was accomplished by an automated custom software script that identified regions with high image intensities that associate with bone, as-well-as regions with low image intensities that associate with air outside the specimen. The image intensities of both these regions were set to match the median tissue intensity, creating a virtual mask of the regions outside the lung for the purpose of deformation error calculations. To reduce the impact of noise, the images were subjected to 2D Gaussian blurring with a radius of 1 .

Using the Drop3d registration tool for motion estimation based on MARKov random fields (cf. http://www.mrf-registration. net/; GlocKer, KomodaKis et al. [26, 38]), 3D deformation fields were calculated by matching the zero pressure dataset to each of the datasets acquired at other pressures. This calculation was accomplished by minimizing the sum of the absolute differences between the voxel intensities of the image datasets.

\subsection{Computation of Strain}

REINHARDT et al. [52] applied an image registration technique to gated CT images of sheep lung from which spatial maps were then constructed whose local Jacobians describe regional volume change. Zhong et al. [71] applied finite element technology to acquire regional volume changes, where 4-noded tetrahedrons were used for voxel discretization from CT images taken of humans with lung cancer.

In this study, discretization is set up in the undeformed configuration using isoparametric 8-noded hexahedral elements, defined from the voxel centroids (Fig. 16). Material particles at the voxel centroids $\mathbb{X}_{a}$ define the initial position of the element nodes as

$$
\mathbb{X}=\sum_{a=1}^{n} N_{a}\left(\xi_{1}, \xi_{2}, \xi_{3}\right) \mathbb{X}_{a}
$$

where $N_{a}$ are standard isoparametric shape functions, and $n$ is the number of nodes or, in our case, voxel centroids. The combination of rigid-body motion and deformation are fully described by the current nodal positions $x_{a}(t)$

$$
x=\sum_{a=1}^{n} N_{a}\left(\xi_{1}, \xi_{2}, \xi_{3}\right) x_{a}
$$

The deformation gradient $\mathbf{F}$, which is the fundamental kinematic quantity for finite deformation, is given as

$$
\mathbf{F}=\sum_{a=1}^{n} x_{a} \otimes \nabla_{0} N_{a},
$$

where $\nabla_{0} N_{a}=\partial N_{a} / \partial \mathbb{X}$ is related to $\nabla_{\xi} N_{a}=$ $\partial N_{a} / \partial \xi$ by the chain rule

$$
\begin{aligned}
\frac{\partial N_{a}}{\partial X} & =\left(\frac{\partial \mathbb{X}}{\partial \xi}\right)^{-T} \frac{\partial N_{a}}{\partial \xi}, \\
\frac{\partial X}{\partial \xi} & =\sum_{a=1}^{n} \mathbb{X}_{a} \otimes \nabla_{\xi} N_{a} .
\end{aligned}
$$

Given a definition for the deformation gradient (Eq. 72), the Finger $\boldsymbol{b}$ and Green $\boldsymbol{C}$ deformation tensors, cf. Freed [18], are easily 
quantified via

$$
\begin{aligned}
\mathrm{b}_{i j} & =\sum_{K=1}^{3} \mathrm{~F}_{i K} \mathrm{~F}_{j K}, \\
\mathrm{C}_{I J} & =\sum_{k=1}^{3} \mathrm{~F}_{k I} \mathrm{~F}_{k J} .
\end{aligned}
$$

From these deformation fields, the Eulerian e and Lagrangian $\boldsymbol{E}$ covariant strain tensors of Almansi [2] and Green [27], both of which measure a change in separation between a pair of neighboring material points, are given by

$$
\begin{aligned}
\mathbf{e} & =\frac{1}{2}\left(\mathbf{I}-\boldsymbol{b}^{-1}\right), \\
\boldsymbol{E} & =\frac{1}{2}(\boldsymbol{C}-\mathbf{I}),
\end{aligned}
$$

while the Eulerian $\boldsymbol{e}$ and Lagrangian $\boldsymbol{E}$ contravariant strain tensors of SigNoRINI [55] and LODGE [43], both of which measure a change in separation between a pair of neighboring material surfaces, are given by

$$
\begin{aligned}
\boldsymbol{e} & =\frac{1}{2}(\boldsymbol{b}-\mathbf{I}), \\
\boldsymbol{E} & =\frac{1}{2}\left(\mathbf{I}-\boldsymbol{C}^{-1}\right) .
\end{aligned}
$$

Volume change is defined simply as

$$
\mathrm{d} V=J \mathrm{~d} V_{0}, \quad J=\operatorname{det} \mathbf{F} .
$$

$J=\mathrm{d} V / \mathrm{d} V_{0}$ is the quantity displayed in Fig. 14.

The fields derived here are applicable for finite deformation analysis.

\subsection{Hysteresis}

In the line drawing of Fig. 14, one readily sees the hysteresis of breathing. This hysteretic area represents the work or cost of breathing; it is the energy expended per breath taken. Hysteresis in the $P V$ curve is a well-known phenomenon that HARRIs [30] attributes to four separate causes: i) recruitment/derecruitment, ii) surfactant, iii) stress relaxation, and $i v$ ) gas absorption.

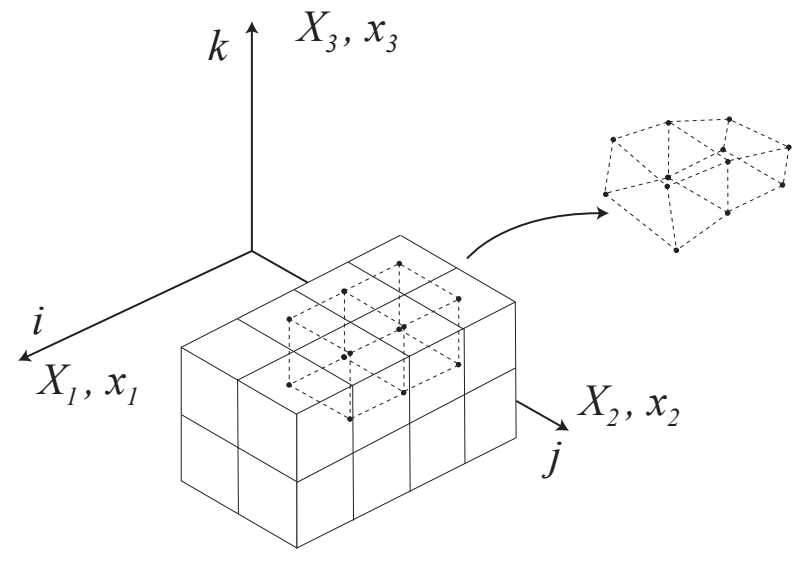

Figure 16: Finite element discretization of an image.

Recruitment can mean two different things in the lung literature. Each has to do with one of the two plateau regions in a typical $S$ shaped $P V$ curve. To the clinician, e.g., [31], recruitment means the engaging of alveoli over the volume of lung as each begins to inflate from its deflated (even possibly collapsed) state at the end of expiration. This mechanism contributes to the low-pressure plateau observed in typical $P V$ curves. To the biomechanician, e.g., [60], recruitment means the engaging of individual collagen fibrils by their straightening from an unloaded crimped state via extension. This enables them to carry load, and thereby stiffens the overall tissue response. This mechanism contributes to the high-pressure plateau observed in typical $P V$ curves. The former is a heterogeneous structural response in that individual alveoli are recruited as if a wave were passing over the lung. The later is a homogeneous material response in that collagen recruitment is an alveolar characteristic.

Surfactant plays an important role in reducing the surface tension of individual alveolar sacs so that they do not collapse when the lungs are deflated. The concentration of surfactant along the air/liquid interface varies over each breath cycle in a complicated physiochemical way [50]. 
The structural constituents of alveoli are predominantly collagen and elastin $[44,56]$ in roughly equal volume fractions [47]. Both are viscoelastic, but with very different characteristic times. The characteristic time of elastin is so short that it basically behaves as a rubbery viscoelastic solid; specifically, as a neo-Hookean material [1]. The characteristic time of collagen, however, is on the same order as normal physiologic processes [11], so its viscoelastic attributes play an important role in lung mechanics.

Lungs are predominantly gas exchangers, with different gases transporting in different directions according to their partial pressures [69]. The pressure within an alveolar sac is therefore subject to the mass rate of exchange in gases via the gas law.

HARris [30] concludes his 2005 review on $P V$ curves by saying:

"If the $P-V$ curve is to become a useful clinical tool, there are still a number of problems to solve. Currently, there is no standard method to obtain the curve or volume history. ... To make matters worse, we still do not understand how alveoli deform during inflation and deflation in human ARDS, so that inferring from a $P-V$ curve what is protecting alveoli or damaging them is exceedingly difficult. ... A bigger issue, perhaps, is that regional mechanical differences are obscured by the $P-V$ curve of the whole lung, and this may be the most important piece of information clinicians need to know. Finally, it is difficult to interpret $P-V$ curves without an absolute measure of lung volume. ... For now it appears that the $P-V$ curve must remain a research tool, or in the clinical situation, reserved for selected patients when it is necessary to try to understand alterations in lung mechanics."

Several of the needs outlined by HARris' are addressed in this research project.

\section{Acknowledgments}

Professor Sally Shepardson from the Biology Department at Saginaw Valley State University is thanked for preparing the samples and then taking the SEM photos of rat lung that appear in this report.

OPENFOAM $\cap$ is a registered trademark of OpenCFD Limited, producer of the OpenFOAM CFD software package.

Data taken from published sources were re-digitized by scanning an image of the required figure, and then digitizing the individual datum points from the scanned image using the g3data software package of Jonas Frantz. http://www. frantz.fi/software/g3data.php.

\section{References}

[1] B. B. Aaron and J. M. Gosline. Elastin as a random-network elastomer: a mechanical and optical analysis of single elastin fibers. Biopolymers, 20:1247-1260, 1981.

[2] E. Almansi. Sulle deformazioni finite dei solidi elastici isotropi. Rendiconti della Reale Accademia dei Lincei: Classe di scienze fisiche, matematiche e naturali, 20:705-714, 1911.

[3] R. E. Brown, J. P. Butler, R. A. Rogers, and D. E. Leith. Mechanical connections between elastin and collagen. Connective Tissue Research, 30:295-308, 1994.

[4] B. Budiansky and E. Kimmel. Elastic moduli of lungs. Journal of Applied Mechanics, 54:351-358, 1987.

[5] R. W. Carton, J. Dainauskas, and J. W. Clark. Elastic properties of single elastic fibers. Journal of Applied Physiology, 17(3):547-551, 1962.

[6] P. J. Dale, F. L. Matthews, and R. C. Schroter. Finite element analysis of lung alveolus. Journal of Biomechanics, 13:865-873, 1980. 
[7] E. Denny and R. C. Schroter. The mechanical behavior of a mammalian lung alveolar duct model. Journal of Biomechanical Engineering, 117:254-261, 1995.

[8] E. Denny and R. C. Schroter. Relationships between alveolar size and fibre distribution in mammalian lung alveolar duct model. Journal of Biomechanical Engineering, 119:289-297, 1997.

[9] E. Denny and R. C. Schroter. Viscoelastic behavior of a lung alveolar duct model. Journal of Biomechanical Engineering, 122:143-151, 2000.

[10] E. Denny and R. C. Schroter. A model of non-uniform lung parenchyma distortion. Journal of Biomechanics, 39:652-663, 2006.

[11] T. C. Doehring, A. D. Freed, E. O. Carew, and I. Vesely. Fractional order viscoelasticity of the aortic valve cusp: an alternative to quasilinear viscoelasticity. Journal of Biomechanical Engineering, 127:700-708, 2005.

[12] D. S. Faffe and W. A. Zin. Lung parenchymal mechanics in health and disease. Physiological Reviews, 89:759-775, 2009.

[13] A. Frankus and G. C. Lee. A theory for distortion studies of lung parenchyma based on alveolar membrane properties. Journal of Biomechanics, 7:101-107, 1974.

[14] J. J. Fredberg and R. D. Kamm. Stress transmission in the lung: pathways from organ to molecule. Annual Review of Physiology, 68:507-541, 2006.

[15] A. D. Freed. Anisotropy in hypoelastic soft-tissue mechanics, I: theory. Journal of Mechanics of Materials and Structures, 3(5):911-928, 2008.

[16] A. D. Freed. Anisotropy in hypoelastic soft-tissue mechanics, II: simple extensional experiments. Journal of Mechanics of Materials and Structures, 4(6):1005-1025, 2009.

[17] A. D. Freed. Hypoelastic soft tissues, part I: theory. Acta Mechanica, 213:189-204, 2010.
[18] A. D. Freed. Soft solids: A primer to the theoretical mechanics of materials. In preparation, 2011.

[19] A. D. Freed and D. R. Einstein. Viscoelastic model for lung parenchyma for multi-scale modeling of respiratory system, Phase I: Hypo-elastic model for CFD implementation. Technical Report PNNL-20340, Pacific Northwest National Laboratory, Richland, WA, May 2011.

[20] A. D. Freed and D. R. Einstein. Hypo-elastic model for lung parenchyma. Biomechanics and Modeling in Mechanobiology, 11:557-573, 2012.

[21] A. D. Freed, D. R. Einstein, and M. S. Sacks. Hypoelastic soft tissues, part II: in-plane biaxial experiments. Acta Mechanica, 213:205-222, 2010.

[22] Y. C. Fung. Elasticity of soft tissues in simple elongation. American Journal of Physiology, 28:1532-1544, 1967.

[23] Y. C. Fung. A model of the lung structure and its validation. Journal of Applied Physiology, 64:2132-2141, 1988.

[24] Y. C. Fung. Biomechanics: Motion, flow, stress, and growth. Springer-Verlag, New York, 1990.

[25] Y. C. Fung. Biomechanics: Circulation. Springer-Verlag, New York, second edition, 1996.

[26] B. Glocker, N. Komodakis, N. Paragios, G. Tziritas, and N. Navab. Inter and intra-modal deformable registration: Continuous deformations meet efficient optimal linear programming. In Information Processing in Medical Imaging, Kerkrade, Netherlands, July 2007.

[27] G. Green. On the propagation of light in crystallized media. Transactions of the Cambridge Philosophical Society, 7:121-140, 1841. 
[28] C. J. Greenshields and H. G. Weller. A unified formulation for continuum mechanics applied to fluid-structure interaction in flexible tubes. International Journal for Numerical Methods in Engineering, 64:1575-1593, 2005.

[29] M. A. Hajji. Indentation of a membrane on an elastic half space. Journal of Applied Mechanics, 45:320-324, 1978.

[30] R. S. Harris. Pressure-volume curves of the respiratory system. Respiratory Care, 50(1):78-98, 2005.

[31] K. G. Hickling. The pressure-volume curve is greatly modified by recruitment, a mathematical model of ARDS lungs. American Journal of Respiratory and Critical Care Medicine, 158:194-202, 1998.

[32] J. Hildebrandt. Comparison of mathematical models for cat lung and viscoelastic balloon derived by Laplace transform methods from pressure-volume data. Bulletin of Mathematical Biophysics, 31(651-667), 1969.

[33] F. G. Hoppin, Jr. and J. Hildebrandt. Mechanical properties of the lung. In J. B. West, editor, Bioengineering Aspects of the Lung, volume 3 of Lung Biology in Health and Disease, pages 83-162. Marcel Dekker, New York, 1977.

[34] J. M. B. Hughes, F. G. Hoppin, Jr., and J. Mead. Effect of lung inflation on bronchial length and diameter in excised lungs. Journal of Applied Physiology, 32(1):25-35, 1972.

[35] A. D. Karakaplan, M. P. Bieniek, and R. Skalak. A mathematical model of lung parenchyma. Journal of Biomechanical Engineering, 102:124-136, 1980.

[36] E. Kimmel and B. Budiansky. Surface tension and the dodecahedron model for lung elasticity. Journal of Biomechanical Engineering, 112:160-167, 1990.

[37] E. Kimmel, R. D. Kamm, and A. H. Shapiro. A cellular model of lung elasticity. Journal of Biomechanical Engineering, 109:126-131, 1987.
[38] N. Komodakis, G. Tziritas, and N. Paragios. Fast, approximately optimal solutions for single and dynamic MRFs. In IEEE Computer Vision and Pattern Recognition, pages 1-8, 2007.

[39] R. Kowe, R. C. Schroter, F. L. Matthews, and D. Hitchings. Analysis of elastic and surface tension effects in the lung alveolus using finite element methods. Journal of Biomechanics, 19(7):541-549, 1986.

[40] S. J. Lai-Fook, T. A. Wilson, R. E. Hyatt, and J. R. Rodarte. Elastic constants of inflated lobes of dog lungs. Journal of Applied Physiology, 40(4):508-513, 1976.

[41] R. K. Lambert and T. A. Wilson. A model for the elastic properties of the lung and their effect on expiratory flow. Journal of Applied Physiology, 34(1):34-48, 1973.

[42] M. A. Lillie and J. M. Gosline. The viscoelastic basis for the tensile strength of elastin. International Journal of Biological Macromolecules, 30:119-127, 2002.

[43] A. S. Lodge. Elastic Liquids: An introductory vector treatment of finite-strain polymer rheology. Academic Press, London, 1964.

[44] M. Matsuda, Y. C. Fung, and S. S. Sobin. Collagen and elastin fibers in human pulmonary alveolar mouths and ducts. Journal of Applied Physiology, 63(3):1185-1194, 1987.

[45] J. Mead. Respiration: pulmonary mechanics. Annual Review of Physiology, 35:169-192, 1973.

[46] M. Mooney. A theory of large elastic deformations. Journal of Applied Physics, 11:582-592, 1940.

[47] E. H. Oldmixon and F. G. Hoppin, Jr. Distribution of elastin and collagen in canine lung alveolar parenchyma. Journal of Applied Physiology, 67(5):1941-1949, 1989.

[48] A. B. Otis. Man's urge to model. Bulletin de Physio-Pathologie Respiratoire, 8:181-198, 1972. 
[49] A. B. Otis. A perspective of respiratory mechanics. Journal of Applied Physiology: Respiratory, Environmental, and Exercise Physiology, 54(5):1183-1187, 1983.

[50] D. R. Otis Jr., E. P. Ingenito, R. D. Kamm, and M. Johnson. Dynamic surface tension of surfactant TA: experiments and theory. Journal of Applied Physiology, 77(6):2681-2688, 1994.

[51] S. F. Perry, A. M. Purohit, S. Boser, I. Mitchell, and F. H. Green. Bronchial casts of human lungs using negative pressure injection. Experimental Lung Research, 26(27-39), 2000.

[52] J. M. Reinhardt, K. Ding, K. Cao, G. E. Christensen, E. A. Hoffman, and S. V. Dodas. Registration-based estimates of local lung tissue expansion compared to xenon CT measures of specific ventilation. Medical Image Analysis, 12:752-763, 2008.

[53] H. T. Robertson, M. A. Krueger, W. J. Lamm, and R. W. Glenny. High-resolution spatial measurements of ventilation-perfusion heterogeneity in rats. Journal of Applied Physiology, 108:1395-1401, 2010.

[54] S. Schürch, M.Lee, and P. Gehr. Pulmonary surfactant: surface properties and function of alveolar and airway surfactant. Pure and Applied Chemsitry, 64(11):1745-1750, 1992.

[55] A. Signorini. Sulle deformazioni thermoelastiche finite. In C. W. Oseen and W. Weibull, editors, Proceedings of the 3rd International Congress for Applied Mechanics, volume 2, pages 80-89, Stockholm, 1930. Ab. Sveriges Litografiska Tryckerier.

[56] S. S. Sobin, Y. C. Fung, and H. M. Tremer. Collagen and elastin fibers in human pulmonary alveolar walls. Journal of Applied Physiology, 64(4):1659-1675, 1988.

[57] D. Stamenović. Micromechanical foundations of pulmonary elasticity. Physiological Reviews, 70(4):1117-1134, 1990.
[58] D. Stamenović and J. C. Smith. Surface forces in lungs. III. Alveolar surface tension and elastic properties of lung parenchyma. Journal of Applied Physiology, 60(4):1358-1362, 1986.

[59] D. Stamenović and D. Yager. Elastic porperties of air- and liquid-filled lung parenchyma. Journal of Applied Physiology, 65(6):2565-2570, 1988.

[60] B. Suki and J. H. T. Bates. Lung tissue mechanics as an emergent phenomenon. Journal of Applied Physiology, 110:1111-1118, 2011.

[61] B. Suki, S. Ito, D. Stamenović, K. R. Lutchen, and E. P. Ingenito. Biomechanics of the lung parenchyma: critical roles of collagen and mechanical forces. Journal of Applied Physiology, 98:1892-1899, 2005.

[62] P. Tchoreloff, A. Gulik, B. Denizot, J. E. Proust, and F. Puisieux. A structural study of interfacial phospholipid and lung surfactant layers by transmission electron microscopy after Blodgett sampling: influence of surface pressure and termperature. Chemistry and Physics of Lipids, 59:151-165, 1991.

[63] L. R. G. Treloar. The Physics of Rubber Elasticity. Clarendon Press, Oxford, third edition, 1975.

[64] C. Truesdell. Hypoelasticity. Journal of Rational Mechanics and Analysis, 4:83-133, 1955.

[65] C. Truesdell. The simplest rate theory of pure elasticity. Communications on Pure and Applied Mathematics, 8:123-132, 1955.

[66] D. J. Tschumperlin, R. Boudreault, and F. Liu. Recent advances and new opportunities in lung mechanobiology. Journal of Biomechanics, 43:99-107, 2010.

[67] D. W. Urry. Physicochemical properties of elastin and constituent peptides, volume 1 of Elastin and Elastases, pages 141-173. CRC Press, Boca Raton, 1989.

[68] E. R. Weibel and J. Gil. Structure-function relationships at the alveolar level. In J. B. 
West, editor, Bioengineering Aspects of the Lung, volume 3 of Lung Biology in Health and Disease, pages 1-81. Marcel Dekker, New York, 1977.

[69] J. B. West. Respiratory Physiology: The essentials. Lippincott Williams \& Wilkins, Philadelphia, eighth edition, 2007.

[70] T. A. Wilson and H. Bachofen. A model for mechanical structure of the alveolar duct. Journal of Applied Physiology, 52(4):1064-1070, 1982.

[71] H. Zhong, J. Jin, M. Ajlouni, B. Movsas, and I. J. Chetty. Measurement of regional compliance using 4DCT images for assessment of radiation treatment. Medical Physics, 38(3):1567-1578, 2011.

\section{A Linear Elements}

There are twenty vertexes in a dodecahedron whose coordinates are most simply expressed as

$$
\begin{aligned}
& ( \pm 1, \pm 1, \pm 1), \\
& (0, \pm 1 / \phi, \pm \phi), \\
& ( \pm 1 / \phi, \pm \phi, 0), \\
& ( \pm \phi, 0, \pm 1 / \phi),
\end{aligned}
$$

when written in terms of a Cartesian reference frame $\left(i^{\prime}, j^{\prime}, \mathbb{k}^{\prime}\right)$ whose origin is located at the center of the dodecahedron. The first set of coordinates associate with an inscribed cube, cf. Fig. 9. The other three sets of coordinates locate the remaining vertexes belonging to the twelve pentagons, with each side having length $L_{0}=2 / \phi$. Parameter $\phi$ is known as the golden ratio, and is defined by

$$
\begin{aligned}
& \phi=\frac{1+\sqrt{5}}{2}=2 \sin (\omega) \approx 1.618 \\
& \frac{1}{\phi}=\frac{2}{1+\sqrt{5}}=\frac{1}{2} \csc (\omega) \approx 0.618 .
\end{aligned}
$$

The dihedral angle (the angle between any two pentagons in a dodecahedron) is $2 \tan ^{-1}(\phi)$.

There are three distinct line elements, viz., $A B$, $B C$, and $C D$ in Fig. 11, with there being ten of each type in the dodecahedron for the mode of deformation being addressed here. The coordinates of locations $A, B, C, D$, and $E$ in this figure, as specified in Eq. (17), do not correspond with the coordinates of Eq. (78). To get the coordinate in Eq. (17), one needs to rotate the coordinates in Eq. (78) via the orthogonal transformation

$$
\left\{\begin{array}{l}
X \\
Y \\
Z
\end{array}\right\}=\left[\begin{array}{ccc}
\cos (\varphi) & 0 & \sin (\varphi) \\
0 & 1 & 0 \\
-\sin (\varphi) & 0 & \cos (\varphi)
\end{array}\right]\left\{\begin{array}{l}
X^{\prime} \\
Y^{\prime} \\
Z^{\prime}
\end{array}\right\}
$$

where $\varphi=\tan ^{-1}(1 / \phi)$ is the angle of rotation (about the $-j^{\prime}$ axis) required to make $\mathbb{k}$ normal to a pentagonal surface, as drawn in Fig. 11. 


\section{A.1 Reference State}

The three, independent, line elements mentioned above are described by three spatial vectors

$$
\begin{aligned}
& \overrightarrow{A B}_{\Omega}=L_{\Omega} \mathbb{U}_{A B}, \\
& \overrightarrow{B C}_{\Omega}=L_{\Omega} \mathbb{U}_{B C}, \\
& \overrightarrow{C D}_{\Omega}=L_{\Omega} \mathbb{U}_{C D},
\end{aligned}
$$

that, in accordance with Eq. (17), can be expressed in terms of three unit vectors with components

$$
\begin{aligned}
\mathbb{U}_{A B} & =\left\{\begin{array}{l}
0 \\
1 \\
0
\end{array}\right\}, \\
\mathbb{U}_{B C} & =\frac{1}{2}\left\{\begin{array}{c}
\cos (\varphi) \\
\tan (\varphi) \\
-2 \cos (\varphi)
\end{array}\right\}, \\
\mathbb{U}_{C D} & =\frac{1}{2}\left\{\begin{array}{c}
\sin (\varphi) \\
-\cot (\varphi) \\
-2 \sin (\varphi)
\end{array}\right\},
\end{aligned}
$$

where $\left\|\mathbb{U}_{A B}\right\|=\left\|\mathbb{U}_{B C}\right\|=\left\|\mathbb{U}_{C D}\right\|=1$, with vector $\mathbb{U}_{A B}$ pointing from $A$ towards $B$, etc.

\section{A.2 Deformed State}

The change in variables introduced in Eq. (20) allows the three, independent, line elements of Eq. (81) to be written in their deformed state as

$$
\begin{aligned}
& \overrightarrow{A B}=L_{\Omega}\left(\mathbb{U}_{A B}+\triangle_{A B}\right), \\
& \overrightarrow{B C}=L_{\Omega}\left(\mathbb{U}_{B C}+\Delta_{B C}\right), \\
& \overrightarrow{C D}=L_{\Omega}\left(\mathbb{U}_{C D}+\Delta_{C D}\right),
\end{aligned}
$$

wherein

$$
\begin{aligned}
& {\Delta_{A B}}=\left\{\begin{array}{c}
0 \\
\left.2 \cos (\omega) y_{2}\right\}, \\
0
\end{array}\right\} \\
& {\Delta_{B C}}=\left\{\begin{array}{c}
\sin (\omega)\left(y_{4}-y_{2}\right) \\
\cos (\omega)\left(y_{4}-y_{2}\right) \\
y_{3}-y_{1}
\end{array}\right\}, \\
& {\Delta_{C D}}=\left\{\begin{array}{c}
(1-\sin (\omega)) y_{4} \\
-\cos (\omega) y_{4} \\
-2 y_{3}
\end{array}\right\},
\end{aligned}
$$

are the nodal displacements caused by traction $\sigma k$.

The stretch in each member relative to state $\left(P_{\Omega}, L_{\Omega}\right)$, e.g., $\lambda_{A B}=L_{A B} / L_{\Omega}$, is quantified by their respective vector norms

$$
\begin{aligned}
& \lambda_{A B}=\left\|\mathbb{U}_{A B}+\triangle_{A B}\right\|, \\
& \lambda_{B C}=\left\|\mathbb{U}_{B C}+\triangle_{B C}\right\|, \\
& \lambda_{C D}=\left\|\mathbb{U}_{C D}+\Delta_{C D}\right\| .
\end{aligned}
$$

However, it is the square of stretch that is used via

$$
\frac{\mathrm{d} L}{L}=\frac{\mathrm{d} \lambda^{2}}{2 \lambda^{2}},
$$

because, as noted by Budiansky \& Kimmel [4], the square of stretch can be expressed as an exact quadratic polynomial of the displacements $y$.

From the above descriptions one can fill out Eq. (21), which expresses the square of stretch as a quadratic equation in $\boldsymbol{y}$. The vectors in Eq. (21) for the three independent stretches have components

$$
\begin{aligned}
& A_{A B}=4 \cos (\omega)\left\{\begin{array}{cccc}
0 & 1 & 0 & 0
\end{array}\right\}^{\mathrm{T}}, \\
& A_{B C}=2 \cos (\varphi)\left\{\begin{array}{c}
1 \\
-1 / \phi \\
-1 \\
1 / \phi
\end{array}\right\}, \\
& A_{C D}=2 \sin (\varphi)\left\{\begin{array}{llll}
0 & 0 & 2 & 1
\end{array}\right\}^{\mathrm{T}},
\end{aligned}
$$

while the matrices in the above expansions have elements

$$
\begin{aligned}
\boldsymbol{A}_{A B}= & {\left[\begin{array}{cccc}
0 & 0 & 0 & 0 \\
0 & 8 \cos ^{2}(\omega) & 0 & 0 \\
0 & 0 & 0 & 0 \\
0 & 0 & 0 & 0
\end{array}\right], } \\
\boldsymbol{A}_{B C}= & {\left[\begin{array}{cccc}
2 & 0 & -2 & 0 \\
0 & 2 & 0 & -2 \\
-2 & 0 & 2 & 0 \\
0 & -2 & 0 & 2
\end{array}\right] } \\
\boldsymbol{A}_{C D}= & {\left[\begin{array}{ccccc}
0 & 0 & 0 & 0 \\
0 & 0 & 0 & 0 \\
0 & 0 & 8 & 0 \\
0 & 0 & 0 & 4(1-\sin (\omega))
\end{array}\right] }
\end{aligned}
$$

The properties of $\phi$, upon which angles $\omega$ and $\varphi$ depend, allow for the simplifications presented in the above components. 


\section{B Areal Elements}

The top and bottom pentagonal areas remain regular and planar during a uniaxial perturbation in loading; however, the ten side pentagons distort from regularity. The following derivation for computing the surface area of a deforming dodecahedron is exact. In the end, the series expansion for areal stretch is truncated to $O\left(y^{3}\right)$ accuracy for use in Eq. (22).

Unlike App. A \& C, which only differ slightly from the formulation of Budiansky \& Kimmel [4], the derivation of this appendix is completely original. Kimmel \& Budiansky [36] used numerical methods to approximate values that are analytically derived herein.

\section{B.1 Regular Pentagons}

With the area of regular pentagon $A B F G H$ in Fig. 11 being given by Eq. (23), it follows that the ratio of its current to reference areas $A / A_{\Omega}$ is equal to the ratio of its characteristic lengths squared $L^{2} / L_{\Omega}^{2}$. Consequently, from Eqs. $(21 \& 22)$, it follows that

$$
\mathbb{B}_{R}=A_{A B} \quad \text { and } \quad \boldsymbol{B}_{R}=\boldsymbol{A}_{A B},
$$

where $A_{A B}$ and $\boldsymbol{A}_{A B}$ are found in Eqs. (87\& 88), respectively.

\section{B.2 Distorted Pentagons}

In order for the series expansion for areal stretching $\Lambda=A / A_{\Omega}$ stated in Eq. (22) to be compatible with the variational bulk response of Eqs. $(9 \& 10)$ whenever $\sigma=0$ and $\boldsymbol{y}=\mathbb{Q}$ for an arbitrary $\delta y$, it follows that

$$
5 \mathbb{B}_{D}+\mathbb{B}_{R}=2\left(A_{A B}+A_{B C}+A_{C D}\right),
$$

so one determines from Eq. (89) that

$$
\mathbb{B}_{D}=\frac{1}{5}\left(A_{A B}+2\left(A_{B C}+A_{C D}\right)\right) .
$$

All that remains to be found is matrix $\boldsymbol{B}_{D}$. Differentiating Eq. (22) twice gives

$$
\boldsymbol{B}_{D}=\frac{1}{A_{\Omega}} \frac{\mathrm{d}^{2} A_{D}}{\mathrm{~d} y \mathrm{~d} y}
$$

reducing the problem to finding the above Hessian. In the analysis that follows, it is determined that $\boldsymbol{B}_{D}$ is itself a quadratic polynomial in $\boldsymbol{y}$.

\section{B.2.1 The Hessian}

The area of a trapezoid is the average length of its two parallel sides times their height of separation. So, for trapezoid $A B C E$, the two parallel sides have reference lengths $A B_{\Omega}=L_{\Omega}$ and $C E_{\Omega}=$ $2 L_{\Omega} \sin (\omega)$ with their height of separation being $H_{\Omega}=L_{\Omega} \cos (\alpha)$, where $\alpha=2 \omega-\pi / 2$. When collected together, the area of this trapezoid is

$$
A_{A B C E_{\Omega}}=L_{\Omega}^{2}(1 / 2+\sin (\omega)) \cos (\alpha),
$$

while the triangle $C D E$ has an area of

$$
A_{C D E_{\Omega}}=L_{\Omega}^{2} \sin (\omega) \cos (\omega),
$$

so that when added they become $\frac{5}{4} L_{\Omega}^{2} \tan (\omega)$, which is the area of each of the 12 regular pentagons of a dodecahedron in its isotropic reference state of $\left(P_{\Omega}, L_{\Omega}\right)$. Recall that $\omega=54^{\circ}$ and $\alpha=18^{\circ}$.

The area $A_{D}$ of pentagon $A B C D E$, distorted by traction $\sigma k$, equates with the area of an isosceles trapezoid $A B C E$, which lies above line $C E$, summed with the area of an isosceles triangle $C D E$, which lies below line $C E$, viz.,

$$
A_{D}=A_{A B C E}+A_{C D E},
$$

that we express as

$$
A_{A B C E}=L_{\Omega}^{2} B H \quad \& \quad A_{C D E}=L_{\Omega}^{2} b h,
$$

where $B$ is the average length of the two parallel edges of the trapezoid with $H$ being its height, while $b$ is the base length of each right triangle (there are two right triangles that make up an isosceles triangle) with $h$ being their height, all of which are scaled against the septal length $L_{\Omega}$ so that

$$
\begin{aligned}
B_{\Omega} & =1 / 2+\sin (\omega), \\
H_{\Omega} & =\cos (\alpha), \\
b_{\Omega} & =\sin (\omega), \\
h_{\Omega} & =\cos (\omega),
\end{aligned}
$$

which describe their reference dimensions. The deformed lengths of these four variables can be 
written as polynomials in the displacement vector $y$; specifically, let

$$
\begin{aligned}
B & =B_{\Omega}+\mathbb{D} \cdot \mathbb{y}, \\
H^{2} & =H_{\Omega}^{2}+\mathbb{E} \cdot \boldsymbol{y}+\frac{1}{2} \boldsymbol{y} \cdot \boldsymbol{E} \mathbb{y}, \\
b & =b_{\Omega}+\mathbb{F} \cdot \boldsymbol{y}, \\
h^{2} & =h_{\Omega}^{2}+\mathbb{G} \cdot \boldsymbol{y}+\frac{1}{2} \boldsymbol{y} \cdot \boldsymbol{G} \mathbb{y},
\end{aligned}
$$

where vectors $\mathbb{D}, \mathbb{E}, \mathbb{F}, \mathbb{G}$ and matrices $\boldsymbol{E}, \boldsymbol{G}$ are all constant valued. It turns out that the base lengths $b$ and $B$ can be written exactly as linear polynomials in $y$; whereas, the heights $h$ and $H$ cannot be expressed as finite polynomials; however, like the stretches, their squares $h^{2}$ and $H^{2}$ can be represented as quadratic polynomials in $y$.

From the above definitions, it follows that the Hessian $\boldsymbol{B}_{D}$ of Eq. (92), in light of Eqs. ( 95 \& 96), is quantified via the following matrix equation

$$
\begin{gathered}
\boldsymbol{B}_{D}=\frac{L_{\Omega}^{2}}{A_{\Omega}}\left(\frac{\mathrm{d} B}{\mathrm{~d} y} \otimes \frac{\mathrm{d} H}{\mathrm{~d} y}+\frac{\mathrm{d} H}{\mathrm{~d} y} \otimes \frac{\mathrm{d} B}{\mathrm{~d} y}\right. \\
+B \frac{\mathrm{d}^{2} H}{\mathrm{~d} y \mathrm{~d} y}+H \frac{\mathrm{d}^{2} B}{\mathrm{~d} y \mathrm{~d} y} \\
+\frac{\mathrm{d} b}{\mathrm{~d} y} \otimes \frac{\mathrm{d} h}{\mathrm{~d} y}+\frac{\mathrm{d} h}{\mathrm{~d} y} \otimes \frac{\mathrm{d} b}{\mathrm{~d} y} \\
\left.+b \frac{\mathrm{d}^{2} h}{\mathrm{~d} y \mathrm{~d} y}+h \frac{\mathrm{d}^{2} b}{\mathrm{~d} y \mathrm{~d} y}\right) .
\end{gathered}
$$

From the polynomial expansions described in Eq. (98), the vector derivatives in Eq. (99) are

$$
\begin{aligned}
\frac{\mathrm{d} B}{\mathrm{~d} y} & =\mathbb{D}, \\
\frac{\mathrm{d} H}{\mathrm{~d} y} & =\frac{1}{2 H}\left(\mathbb{E}+E_{\mathbb{y}}\right), \\
\frac{\mathrm{d} b}{\mathrm{~d} y} & =\mathbb{F}, \\
\frac{\mathrm{d} h}{\mathrm{~d} y} & =\frac{1}{2 h}\left(\mathbb{G}+G_{\mathbb{y}}\right),
\end{aligned}
$$

while the matrix derivatives in Eq. (99) are

$$
\begin{aligned}
& \frac{\mathrm{d}^{2} B}{\mathrm{~d} y \mathrm{~d} y}=\mathbf{0}, \\
& \frac{\mathrm{d}^{2} H}{\mathrm{~d} y \mathrm{~d} y}=\frac{1}{2 H}(\boldsymbol{E} \\
& \left.-\frac{1}{2 H^{2}}\left((\mathbb{E}+\boldsymbol{E} y) \otimes\left(\mathbb{E}+\boldsymbol{E}_{y}\right)\right)\right), \\
& \frac{\mathrm{d}^{2} b}{\mathrm{~d} y \mathrm{~d} y}=\mathbf{0}, \\
& \frac{\mathrm{d}^{2} h}{\mathrm{~d} y \mathrm{~d} y}=\frac{1}{2 h}(\boldsymbol{G} \\
& \left.-\frac{1}{2 h^{2}}\left((\mathbb{G}+\boldsymbol{G} y) \otimes\left(\mathbb{G}+\boldsymbol{G}_{y}\right)\right)\right),
\end{aligned}
$$

where the chain rule has been used.

The above formulation for $\boldsymbol{B}_{D}$ is a quadratic function in $y$ whose value at $y=\mathbb{Q}$ is

$$
\begin{aligned}
\left.\boldsymbol{B}_{D}\right|_{y=\mathbb{D}} & =\frac{L_{\Omega}^{2}}{A_{\Omega}}\left\{\frac{1}{2 H_{\Omega}}(\mathbb{D} \otimes \mathbb{E}+\mathbb{E} \otimes \mathbb{D})\right. \\
& +\frac{B_{\Omega}}{2 H_{\Omega}}\left(\boldsymbol{E}-\frac{1}{2 H_{\Omega}^{2}} \mathbb{E} \otimes \mathbb{E}\right) \\
& +\frac{1}{2 h_{\Omega}}(\mathbb{F} \otimes \mathbb{G}+\mathbb{G} \otimes \mathbb{F}) \\
& \left.+\frac{b_{\Omega}}{2 h_{\Omega}}\left(\boldsymbol{G}-\frac{1}{2 h_{\Omega}^{2}} \mathbb{G} \otimes \mathbb{G}\right)\right\} .
\end{aligned}
$$

What remains to quantify are the four vectors and two matrices present in the above expression, see Eqs. (107 \& 110-114).

\section{B.2.2 Vectors \& Matrices of Deformation}

From the reference coordinates (17) and their displacements (18), one determines that the base lengths referred to in Eq. (96) for the deformed $A B C E$ trapezoid and $C D E$ triangle have dimensionless lengths of

$$
\begin{aligned}
B & =B_{\Omega}+\cos (\omega)\left(y_{2}+y_{4}\right), \\
b & =b_{\Omega}+\cos (\omega) y_{4},
\end{aligned}
$$


with squared heights of

$$
\begin{aligned}
H^{2} & =\left(\frac{1}{2} \cos (\varphi)+\frac{1}{2} \phi\left(y_{4}-y_{2}\right)\right)^{2} \\
& +\left(\cos (\varphi)+y_{1}-y_{3}\right)^{2}, \\
h^{2} & =\left(\frac{1}{2} \sin (\varphi)+(1-\phi / 2) y_{4}\right)^{2} \\
& +\left(\sin (\varphi)+2 y_{3}\right)^{2},
\end{aligned}
$$

which are most easily envisioned by projecting the $A B C D E$ surface onto the $i \wedge k$ plane, where it becomes a segmented line.

Expanding the above expressions according to their definitions allows the components of the above defined vectors and matrices to be quantified. Vector $\mathbb{D}$, which belongs to the trapezoidal base length $B$ of Eq. (98), has components

$$
\mathbb{D}=\left\{\begin{array}{c}
0 \\
\cos (\omega) \\
0 \\
\cos (\omega)
\end{array}\right\} .
$$

Vector $\mathbb{E}$ and matrix $\boldsymbol{E}$, which belong to the trapezoidal height squared $H^{2}$ of Eq. (98), have components

$$
\mathbb{E}=\cos (\varphi)\left\{\begin{array}{c}
2 \\
-\phi / 2 \\
-2 \\
\phi / 2
\end{array}\right\}
$$

and

$$
\boldsymbol{E}=\left[\begin{array}{cccc}
2 & 0 & -2 & 0 \\
0 & \phi^{2} / 2 & 0 & -\phi^{2} / 2 \\
-2 & 0 & 2 & 0 \\
0 & -\phi^{2} / 2 & 0 & \phi^{2} / 2
\end{array}\right]
$$

Vector $\mathbb{F}$, which belongs to base length $b$ for the right triangle in Eq. (98), has components

$$
\mathbb{F}=\left\{\begin{array}{c}
0 \\
0 \\
0 \\
\cos (\omega)
\end{array}\right\}
$$

And vector $\mathbb{G}$ and matrix $\boldsymbol{G}$, which belong to the square of this triangle's height $h^{2}$ via Eq. (98), have components

$$
\mathbb{G}=\sin (\varphi)\left\{\begin{array}{c}
0 \\
0 \\
4 \\
1-\phi / 2
\end{array}\right\},
$$

and

$$
\boldsymbol{G}=\left[\begin{array}{cccc}
0 & 0 & 0 & 0 \\
0 & 0 & 0 & 0 \\
0 & 0 & 8 & 0 \\
0 & 0 & 0 & 2(1-\phi / 2)^{2}
\end{array}\right]
$$

Inserting Eqs. (23, 97 \& 105-110) into Eq. (102) allows matrix $\boldsymbol{B}_{D}$ in Eq. (22) to be quantified. The nature of the vector outer products that are involved are such that a simple final expression is not to be had. Nevertheless, the symmetric forms of these outer products can be substantially simplified due to the properties of the golden ratio $\phi$, specifically

$$
\begin{aligned}
& \frac{1}{2}(\mathbb{D} \otimes \mathbb{E}+\mathbb{E} \otimes \mathbb{D})= \\
& \frac{1}{2}\left[\begin{array}{cccc}
0 & 1 & 0 & 1 \\
1 & -\phi / 2 & -1 & 0 \\
0 & -1 & 0 & -1 \\
1 & 0 & -1 & \phi / 2
\end{array}\right],
\end{aligned}
$$

$$
\begin{aligned}
& \frac{1}{2}(\mathbb{E} \otimes \mathbb{E})= \frac{\cos ^{2}(\varphi)}{2} \\
& \times\left[\begin{array}{cccc}
4 & -\phi & -4 & \phi \\
-\phi & \phi^{2} / 4 & \phi & -\phi^{2} / 4 \\
-4 & \phi & 4 & -\phi \\
\phi & -\phi^{2} / 4 & -\phi & \phi^{2} / 4
\end{array}\right],
\end{aligned}
$$

$$
\begin{aligned}
\frac{1}{2}(\mathbb{F} \otimes \mathbb{G}+\mathbb{G} \otimes \mathbb{F})= \\
\frac{1}{2}\left[\begin{array}{cccc}
0 & 0 & 0 & 0 \\
0 & 0 & 0 & 0 \\
0 & 0 & 0 & 2 / \phi \\
0 & 0 & 2 / \phi & 1 / \phi-1 / 2
\end{array}\right],
\end{aligned}
$$

and

$$
\begin{aligned}
\frac{1}{2}(\mathbb{G} \otimes \mathbb{G}) & =\frac{\sin ^{2}(\varphi)}{2} \\
\times & {\left[\begin{array}{cccc}
0 & 0 & 0 & 0 \\
0 & 0 & 0 & 0 \\
0 & 0 & 16 & 4(1-\phi / 2) \\
0 & 0 & 4(1-\phi / 2) & (1-\phi / 2)^{2}
\end{array}\right] . }
\end{aligned}
$$




\section{Volume Elements}

The distorted dodecahedron is segmented into a collection of tetrahedra for computing its volume. Given vertexes described by position vectors $a, \not{b}$ and $\mathfrak{c}$, with the forth vertex being located at the origin, the volume of a tetrahedron is proportional to its scalar triple product, viz., $\left.V=\frac{1}{6} \mid a \cdot(b) \times c\right) \mid$, which describes a cubic polynomial in $y$. Care must be taken to change the sign whenever the scalar triple product yields a negative number. This is most easily checked at $\boldsymbol{y}=\mathbb{D}$. All total, we decompose the distorted dodecahedron into seventy individual tetrahedra that belong to four shape types.

In order for the series expansion for volumetric stretching $\Delta=V / V_{\Omega}$ stated in Eq. (24) to be compatible with the variational bulk response of Eqs. ( $9 \& 10)$ whenever $\sigma=0$ and $y=\mathbb{Q}$ for an arbitrary $\delta y$, it follows from Eq. (21) that

$$
\mathbb{C}=\frac{1}{2}\left(A_{A B}+A_{B C}+A_{C D}\right),
$$

which agrees (in numeric value) with the equivalent $\mathbb{C}$ vector derived by Budiansky \& Kimmel [4]. This is the linear coefficient for $y$ in Eq. (24). We now seek the quadratic coefficient that is matrix $\boldsymbol{C}$.

A single tetrahedron can be used to describe the volumes whose outer surfaces associate with the regular pentagons on the upper and lower faces, there being ten associated tetrahedra overall. The quadratic term in the volume expansion for this grouping reduces to

$$
\frac{5}{3} L_{\Omega}^{3}\left(\phi y_{1} y_{2}+\frac{1}{4} \phi^{3} y_{2}^{2}\right)
$$

The triangular face used to compute the volume of a tetrahedron from this grouping has corners located at $A B W$, where $A$ and $B$ are quantified in Eq. (17), while $W$ has coordinates $L_{\Omega}\left(0,0, \phi^{2} \cos (\varphi) / 2\right)$ in the reference state $\left(P_{\Omega}, L_{\Omega}\right)$, cf. Fig. 11 .

The $C D E$ isosceles triangle is made up of two right triangles of equal area. There are 20 such triangles on the surface of a dodecahedron. The twenty tetrahedra that associate with these triangular faces have a quadratic term in their volume expansion of

$$
\frac{5}{3} L_{\Omega}^{3}\left(\phi(2+\phi) y_{3} y_{4}+\frac{1}{4}(1+2 / \phi) y_{4}^{2}\right) .
$$

The right triangle used to compute the volume of a tetrahedron from this grouping has corners $C D X$, where $C$ and $D$ are quantified in Eq. (17), while $X$ has coordinates $L_{\Omega}\left(\phi^{2} \cos (\varphi) / 2,0, \sin (\varphi) / 2\right)$ in the reference state $\left(P_{\Omega}, L_{\Omega}\right)$.

The $A B C E$ isosceles trapezoid is made up of three triangles. The outer two triangles, $A X E$ and $B C X$, are equal in area due to symmetry. The inner triangle $A B X$ can be further divided into two right triangles of equal area, viz., $A Y X$ and $B X Y$. There are 20 of these outer triangles that appear on the surface of a dodecahedron. The volume of these twenty tetrahedra has a quadratic term in its series expansion of

$$
\begin{aligned}
\frac{5}{3} L_{\Omega}^{3} & \left(\phi^{2} y_{1} y_{4}-\frac{1}{2} \phi^{2} y_{2} y_{3}\right. \\
& \left.\quad-\frac{1}{4} y_{2} y_{4}-\frac{1}{2} \phi y_{3} y_{4}+\frac{1}{4} \phi^{3} y_{4}^{2}\right) .
\end{aligned}
$$

There are also 20 such inner right triangles that appear on the face of a dodecahedron. The volume of these twenty tetrahedra has a quadratic term in its series expansion of

$$
\begin{aligned}
\frac{5}{3} L_{\Omega}^{3} & \left(\frac{1}{2} \phi^{2} y_{1} y_{2}+\frac{1}{2} \phi y_{1} y_{4}\right. \\
& \left.-\frac{1}{4} y_{2}^{2}-\phi y_{2} y_{3}+\frac{1}{4} \phi^{3} y_{2} y_{4}\right)
\end{aligned}
$$

$Y$ has coordinates $L_{\Omega}\left(\phi^{2} \sin (\varphi) / 2,0, \cos (\varphi) / 2\right)$ in the reference state $\left(P_{\Omega}, L_{\Omega}\right)$.

Summing the above expressions, which collectively describe the quadratic contribution to volume change in a uniaxially distorted dodecahedron when normalizing by the reference volume $V_{\Omega}$ given in Eq. (25), leads to the following expression for matrix $C$ in Eq. (24), i.e.,

$$
\begin{gathered}
C=\frac{2}{3 \tan ^{2}(\omega)} \\
\times\left[\begin{array}{cccc}
0 & 1+\phi / 2 & 0 & 1 / 2+\phi \\
1+\phi / 2 & 1 & -(1+\phi / 2) & 1 / 2 \\
0 & -(1+\phi / 2) & 0 & 3 / 2+\phi \\
1 / 2+\phi & 1 / 2 & 3 / 2+\phi & 2
\end{array}\right] .
\end{gathered}
$$

This matrix is slightly different from the analogous one derived by Budiansky \& Kimmel [4]; however, the constant and linear terms in our series expansions for $\Delta=V / V_{\Omega}$, cf. Eq. (24), are the same. 
The fact that our formulations differ in their quadratic contributions is not unexpected, because of the different assumptions imposed on the shape of the deformed dodecahedron. They represented each pentagonal face distorted from regularity with five planar triangles sharing a common center node whose displacement is the mean of the pentagon's vertexes; whereas, we represent each distorted pentagonal face with a planar isosceles triangle and a planar isosceles trapezoid sharing a common edge.

\section{Geometric Identities}

The geometry of a dodecahedron can be expressed in terms of the golden ratio $\phi$ of Eq. (79), cf. App. A. There are numerous identities between this ratio and the various angles that can be used to describe a dodecahedron, some of which are cataloged below.

$$
\begin{aligned}
& \sin (\omega)=\phi / 2, \\
& \tan (\varphi)=1 / \phi, \\
& \cos (\varphi) \cos (\omega)=1 / 2, \\
& \phi \sin (\varphi) \cos (\omega)=1 / 2, \\
& \phi^{2} \cos ^{2}(\varphi)-\sin ^{2}(\varphi)=\phi, \\
& 2 \tan (\omega)(1-\sin (\omega))=\sin (\varphi), \\
& \phi^{2}-1 / \phi=2, \\
& \phi^{2}+1 / \phi+2 / \phi^{2}=4 .
\end{aligned}
$$

